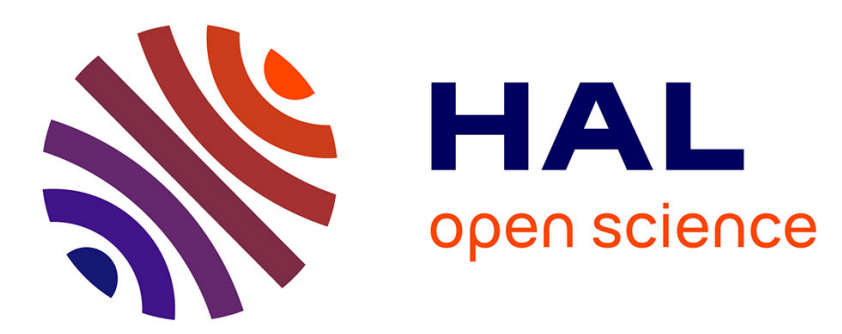

\title{
Convergence of damped inertial dynamics governed by regularized maximally monotone operators
}

\author{
Hedy Attouch, Alexandre Cabot
}

\section{To cite this version:}

Hedy Attouch, Alexandre Cabot. Convergence of damped inertial dynamics governed by regularized maximally monotone operators . Journal of Differential Equations, 2018, 264 (12), pp.7138-7182. 10.1016/j.jde.2018.02.017 . hal-01648383v2

\section{HAL Id: hal-01648383 \\ https://hal.science/hal-01648383v2}

Submitted on 7 Apr 2018

HAL is a multi-disciplinary open access archive for the deposit and dissemination of scientific research documents, whether they are published or not. The documents may come from teaching and research institutions in France or abroad, or from public or private research centers.
L'archive ouverte pluridisciplinaire HAL, est destinée au dépôt et à la diffusion de documents scientifiques de niveau recherche, publiés ou non, émanant des établissements d'enseignement et de recherche français ou étrangers, des laboratoires publics ou privés. 


\title{
CONVERGENCE OF DAMPED INERTIAL DYNAMICS GOVERNED BY REGULARIZED MAXIMALLY MONOTONE OPERATORS
}

\author{
HEDY ATTOUCH AND ALEXANDRE CABOT
}

\begin{abstract}
In a Hilbert space setting, we study the asymptotic behavior, as time $t$ goes to infinity, of the trajectories of a second-order differential equation governed by the Yosida regularization of a maximally monotone operator with time-varying positive index $\lambda(t)$. The dissipative and convergence properties are attached to the presence of a viscous damping term with positive coefficient $\gamma(t)$. A suitable tuning of the parameters $\gamma(t)$ and $\lambda(t)$ makes it possible to prove the weak convergence of the trajectories towards zeros of the operator. When the operator is the subdifferential of a closed convex proper function, we estimate the rate of convergence of the values. These results are in line with the recent articles by Attouch-Cabot [3], and Attouch-Peypouquet [8]. In this last paper, the authors considered the case $\gamma(t)=\frac{\alpha}{t}$, which is naturally linked to Nesterov's accelerated method. We unify, and often improve the results already present in the literature.
\end{abstract}

Key words: asymptotic stabilization; damped inertial dynamics; Lyapunov analysis; maximally monotone operators; time-dependent viscosity; Yosida regularization.

AMS subject classification. 37N40, 46N10, 49M30, 65K05, 65K10, 90C25.

\section{INTRODUCTION}

Throughout this paper, $\mathcal{H}$ is a real Hilbert space endowed with the scalar product $\langle.,$.$\rangle and the$ corresponding norm $\|$.$\| . Let A: \mathcal{H} \rightarrow 2^{\mathcal{H}}$ be a maximally monotone operator. Given continuous functions $\gamma:\left[t_{0},+\infty\left[\rightarrow \mathbb{R}_{+}\right.\right.$and $\lambda:\left[t_{0},+\infty\left[\rightarrow \mathbb{R}_{+}^{*}\right.\right.$ where $t_{0}$ is a fixed real number, we consider the second-order evolution equation

$(\operatorname{RIMS})_{\gamma, \lambda} \quad \ddot{x}(t)+\gamma(t) \dot{x}(t)+A_{\lambda(t)}(x(t))=0, \quad t \geq t_{0}$,

where

$$
A_{\lambda}=\frac{1}{\lambda}\left(I-(I+\lambda A)^{-1}\right)
$$

is the Yosida regularization of $A$ of index $\lambda>0$ (see Appendix A.1 for its main properties). The terminology (RIMS) $\gamma, \lambda$ is a shorthand for "Regularized Inertial Monotone System" with parameters $\gamma, \lambda$. Thanks to the Lipschitz continuity properties of the Yosida approximation, this system falls within the framework of the Cauchy-Lipschitz theorem, which makes it a well-posed system for arbitrary Cauchy data. The above system involves two time-dependent positive parameters: the damping parameter $\gamma(t)$, and the Yosida regularization parameter $\lambda(t)$. We shall see that, under a suitable tuning of the parameters $\gamma(t)$ and $\lambda(t)$, the trajectories of (RIMS) ${ }_{\gamma, \lambda}$ converge to solutions of the monotone inclusion

$$
0 \in A(x) \text {. }
$$

Indeed, the design of rapidly convergent dynamics and algorithms to solve monotone inclusions is a difficult problem of fundamental importance in many domains: optimization, equilibrium theory, economics and game theory, partial differential equations, statistics, among other subjects. Trajectories of

Date: February 19, 2018. 
$(\text { RIMS })_{\gamma, \lambda}$ do so in a robust manner. Indeed, when $A$ is the subdifferential of a closed convex proper function $\Phi: \mathcal{H} \rightarrow \mathbb{R} \cup\{+\infty\}$, we will obtain rates of convergence of the values, which are comparable to the accelerated method of Nesterov. With this respect, as a main advantage of our approach, we can handle nonsmooth functions $\Phi$.

1.1. Introducing the dynamics. The (RIMS) ${ }_{\gamma, \lambda}$ system is a natural development of some recent studies concerning rapid inertial dynamics for convex optimization and monotone equilibrium problems. We will rely heavily on the techniques developed in [3] concerning the general damping coefficient $\gamma(t)$, and in [8] concerning the general Yosida regularization parameter $\lambda(t)$.

1.1.1. General damping coefficient $\gamma(t)$. Some simple observations lead to the introduction of quantities that play a central role in our analysis. Taking $A=0$, then $A_{\lambda}=0$, and (RIMS) ${ }_{\gamma, \lambda}$ boils down to the linear differential equation

$$
\ddot{x}(t)+\gamma(t) \dot{x}(t)=0 .
$$

Let us multiply this equality by the integrating factor

$$
p(t)=e^{\int_{t_{0}}^{t} \gamma(\tau) d \tau}
$$

and integrate on $\left[t_{0}, t\right]$. We obtain $p(t) \dot{x}(t)=\dot{x}\left(t_{0}\right)$ for every $t \geq t_{0}$. By integrating again, we find

$$
x(t)=x\left(t_{0}\right)+\left(\int_{t_{0}}^{t} \frac{d s}{p(s)}\right) \dot{x}\left(t_{0}\right)
$$

It ensues immediately that the trajectory $x($.$) converges if and only if \dot{x}\left(t_{0}\right)=0$ or

$$
\int_{t_{0}}^{+\infty} \frac{d s}{p(s)}<+\infty
$$

Throughout the paper, we always assume that condition $\left(H_{0}\right)$ is satisfied. For $s \geq t_{0}$, we then define the quantity $\Gamma(s)$ by

$$
\Gamma(s)=\left(\int_{s}^{+\infty} \frac{d u}{p(u)}\right) p(s) .
$$

The function $s \mapsto \Gamma(s)$ plays a key role in the asymptotic behavior of the trajectories of $(\text { RIMS })_{\gamma, \lambda}$. This was brought to light by the authors in the potential case, see [3] (no regularization process was used in this work). The theorem below gathers the main results obtained in [3] for a gradient operator $A=\nabla \Phi$. It enlights the basic assumptions on the function $\gamma(t)$ which give rates of convergence of the values.

Theorem (Attouch and Cabot [3]). Let $\Phi: \mathcal{H} \rightarrow \mathbb{R}$ be a convex function of class $\mathcal{C}^{1}$ such that $\operatorname{argmin} \Phi \neq \emptyset$. Let us assume that $\gamma:\left[t_{0},+\infty\left[\rightarrow \mathbb{R}_{+}\right.\right.$is a continuous function satisfying:

(i) $\int_{t_{0}}^{+\infty} \frac{d s}{p(s)}<+\infty$;

(ii) There exist $t_{1} \geq t_{0}$ and $m<\frac{3}{2}$ such that $\gamma(t) \Gamma(t) \leq m$ for every $t \geq t_{1}$;

(iii) $\int_{t_{0}}^{+\infty} \Gamma(s) d s=+\infty$.

Then every solution trajectory $x:\left[t_{0},+\infty[\rightarrow \mathcal{H}\right.$ of

$$
\ddot{x}(t)+\gamma(t) \dot{x}(t)+\nabla \Phi(x(t))=0,
$$

converges weakly toward some $x^{*} \in \operatorname{argmin} \Phi$, and satisfies the following rates of convergence:

$$
\Phi(x(t))-\min _{\mathcal{H}} \Phi=o\left(\frac{1}{\int_{t_{0}}^{t} \Gamma(s) d s}\right) \quad \text { and } \quad\|\dot{x}(t)\|=o\left(\frac{1}{\int_{t_{0}}^{t} \Gamma(s) d s}\right)^{1 / 2} \quad \text { as } t \rightarrow+\infty .
$$


The (IGS) $)_{\gamma}$ system was previously studied by Cabot, Engler and Gadat $[17,18]$ in the case of a vanishing damping coefficient $\gamma(t)$ and for a possibly nonconvex potential $\Phi$. The importance of the dynamics $(\mathrm{IGS})_{\gamma}$ in the case $\gamma(t)=\alpha / t(\alpha>1)$ was highlighted by Su, Boyd and Candés in [28]. They showed that taking $\alpha=3$ gives a continuous version of the accelerated gradient method of Nesterov. The corresponding rate of convergence for the values is at most of order $\mathcal{O}\left(1 / t^{2}\right)$ as $t \rightarrow+\infty$. Let us show how this result can be obtained as a consequence of the above general theorem. Indeed, taking $\gamma(t)=\alpha / t$ gives after some elementary computation

$$
\Gamma(t)=\left(\frac{t}{t_{0}}\right)^{\alpha}\left[\int_{t}^{+\infty}\left(\frac{t_{0}}{\tau}\right)^{\alpha} d \tau\right]=t^{\alpha}\left[\frac{\tau^{-\alpha+1}}{-\alpha+1}\right]_{t}^{+\infty}=\frac{t}{\alpha-1} .
$$

Then, the condition $\gamma(t) \Gamma(t) \leq m$ with $m<\frac{3}{2}$ is equivalent to $\alpha>3$. As a consequence, for $\gamma(t)=\alpha / t$ and $\alpha>3$, we obtain the convergence of the trajectories of (IGS) $\gamma$ and the rates of convergence

$$
\Phi(x(t))-\min _{\mathcal{H}} \Phi=o\left(\frac{1}{t^{2}}\right) \quad \text { and } \quad\|\dot{x}(t)\|=o\left(\frac{1}{t}\right) \quad \text { as } t \rightarrow+\infty .
$$

This result was first established in [4] and [22]. Because of its importance, a rich literature has been devoted to the algorithmic versions of these results, see $[4,7,12,19,28]$ and the references therein. The above theorem relies on energetical arguments that are not available in the general framework of monotone operators. It ensues that the expected results in this context are weaker than in the potential case, and require different techniques. That's where the Yosida regularization comes into play.

1.1.2. General regularization parameter $\lambda(t)$. Our approach is in line with Attouch and Peypouquet [8] who studied the system (RIMS) ${ }_{\gamma, \lambda}$ with a general maximally monotone operator, and in the particular case $\gamma(t)=\alpha / t$ (the importance of this system has been stressed just above). This approach can be traced back to Álvarez-Attouch [1] and Attouch-Maingé [6] who studied the equation

$$
\ddot{x}(t)+\gamma \dot{x}(t)+A(x(t))=0,
$$

where $A$ is a cocoercive operator. Several variants of the above equation were considered by Bot and Csetnek (see [13] for the case of a time-dependent coefficient $\gamma(t)$, and [14] for a linear anisotropic damping). Cocoercivity plays an important role, not only to ensure the existence of solutions, but also in analyzing their long-term behavior. Attouch-Maingé [6] proved the weak convergence of the trajectories to zeros of $A$ if the cocoercivity parameter $\lambda$ and the damping coefficient $\gamma$ satisfy the condition $\lambda \gamma^{2}>1$. Taking into account that for $\lambda>0$, the operator $A_{\lambda}$ is $\lambda$-cocoercive and that $A_{\lambda}^{-1}(0)=A^{-1}(0)$ (see Appendix A.1), we immediately deduce that, under the condition $\lambda \gamma^{2}>1$, each trajectory of

$$
\ddot{x}(t)+\gamma \dot{x}(t)+A_{\lambda}(x(t))=0
$$

converges weakly to a zero of $A$. In the quest for a faster convergence, in the case $\gamma(t)=\alpha / t$, AttouchPeypouquet introduced a time-dependent regularizing parameter $\lambda(\cdot)$ satisfying

$$
\lambda(t) \times \frac{\alpha^{2}}{t^{2}}>1
$$

for $t \geq t_{0}$. So doing, in the case of a general maximal monotone operator, they were able to prove the asymptotic convergence of the trajectories to zeros of $A$. Our approach will consist in extending these results to the case of a general damping coefficient $\gamma(t)$, taking advantage of the techniques developed in the above mentioned papers [3] and [8]. 
1.2. Organization of the paper. The paper is divided into three parts. Part A concerns a general maximally monotone operator $A$. We show that a suitable tuning of the damping parameter and of the Yosida regularization parameter, gives the weak convergence of the trajectories. Then, we specialize our results to some important cases, including the case of the continuous version of the Nesterov method, that is, $\gamma(t)=\frac{\alpha}{t}$. In part B, we examine the ergodic convergence properties of the trajectories. In part C, we consider the case where $A$ is the subdifferential of a closed convex proper function $\Phi: \mathcal{H} \rightarrow \mathbb{R} \cup\{+\infty\}$. In this case, we will obtain rates of convergence of the values. In the Appendix we have collected several lemmas related to Yosida's approximation, to Moreau's envelopes and to the study of scalar differential inequalities that play a central role in the Lyapunov analysis of our system.

\section{PART A: DYNAMICS FOR A GENERAL MAXIMALLY MONOTONE OPERATOR}

In this part, $A: \mathcal{H} \rightarrow 2^{\mathcal{H}}$ is a general maximally monotone operator such that zer $A \neq \emptyset$, and $t_{0}$ is a fixed real number.

\section{Convergence Results}

Let us first establish the existence and uniqueness of a global solution to the Cauchy problem associated with equation $(\mathrm{RIMS})_{\gamma, \lambda}$.

Proposition 2.1. Let $A: \mathcal{H} \rightarrow 2^{\mathcal{H}}$ be a maximally monotone operator, and let $\gamma:\left[t_{0},+\infty\left[\rightarrow \mathbb{R}_{+}\right.\right.$and $\lambda:\left[t_{0},+\infty\left[\rightarrow \mathbb{R}_{+}^{*}\right.\right.$ be continuous functions. Then, for any $x_{0} \in \mathcal{H}, v_{0} \in \mathcal{H}$, there exists a unique global solution $x \in \mathcal{C}^{2}\left(\left[t_{0},+\infty[, \mathcal{H})\right.\right.$ to equation $(\mathrm{RIMS})_{\gamma, \lambda}$, satisfying the initial conditions $x\left(t_{0}\right)=x_{0}$ and $\dot{x}\left(t_{0}\right)=v_{0}$.

Proof. The argument is standard and consists in writing $(\mathrm{RIMS})_{\gamma, \lambda}$ as a first-order system in $\mathcal{H} \times \mathcal{H}$. By setting

$$
X(t)=\left(\begin{array}{c}
x(t) \\
\dot{x}(t)
\end{array}\right) \quad \text { and } \quad F(t, u, v)=\left(\begin{array}{c}
v \\
-\gamma(t) v-A_{\lambda(t)}(u)
\end{array}\right)
$$

equation $(\mathrm{RIMS})_{\gamma, \lambda}$ amounts to the first-order differential system $\dot{X}(t)=F(t, X(t))$. Owing to the $\frac{1}{\lambda}$-Lipschitz continuity of $A_{\lambda}$, one can easily check that the conditions of the global Cauchy-Lipschitz theorem are satisfied. The reader is referred to [20, Proposition 6.2.1] for such a global non-autonomous version of the Cauchy-Lipschitz theorem.

To establish the weak convergence of the trajectories of $(\mathrm{RIMS})_{\gamma, \lambda}$, we will apply Opial lemma [24], that we recall in its continuous form.

Lemma 2.2 (Opial). Let $S$ be a nonempty subset of $\mathcal{H}$, and let $x:\left[t_{0},+\infty[\rightarrow \mathcal{H}\right.$. Assume that

(i) for every $z \in S, \lim _{t \rightarrow+\infty}\|x(t)-z\|$ exists;

(ii) every weak sequential limit point of $x(t)$, as $t \rightarrow+\infty$, belongs to $S$.

Then $x(t)$ converges weakly as $t \rightarrow+\infty$ to a point in $S$.

We associate to the continuous function $\gamma:\left[t_{0},+\infty\left[\rightarrow \mathbb{R}_{+}\right.\right.$the function $p:\left[t_{0},+\infty\left[\rightarrow \mathbb{R}_{+}^{*}\right.\right.$ given by $p(t)=e^{\int_{t_{0}}^{t} \gamma(\tau) d \tau}$ for every $t \geq t_{0}$. Under assumption $\left(H_{0}\right)$, the function $\Gamma:\left[t_{0},+\infty\left[\rightarrow \mathbb{R}_{+}^{*}\right.\right.$ is then defined

by $\Gamma(s)=\left(\int_{s}^{+\infty} \frac{d u}{p(u)}\right) p(s)$ for every $s \geq t_{0}$. Besides the function $\Gamma$, to analyze the asymptotic behavior of the trajectory of the system (RIMS) ${ }_{\gamma, \lambda}$ we will also use the quantity $\Gamma(s, t)$, which is defined by, for any $s, t \in\left[t_{0},+\infty[\right.$,

$$
\Gamma(s, t)=\left(\int_{s}^{t} \frac{d u}{p(u)}\right) p(s) \quad \text { if } s \leq t, \text { and } \Gamma(s, t)=0 \text { if } s>t .
$$


For each $s \in\left[t_{0},+\infty[\right.$, the quantity $\Gamma(s, t)$ tends increasingly toward $\Gamma(s)$ as $t \rightarrow+\infty$. The monotone convergence theorem then implies that

$$
\lim _{t \rightarrow+\infty} \int_{t_{0}}^{t} \Gamma(s, t) d s=\lim _{t \rightarrow+\infty} \int_{t_{0}}^{+\infty} \Gamma(s, t) d s=\int_{t_{0}}^{+\infty} \Gamma(s) d s,
$$

since $\Gamma(s, t)=0$ for $s \geq t$. Let us state the main result of this section.

Theorem 2.3. Let $A: \mathcal{H} \rightarrow 2^{\mathcal{H}}$ be a maximally monotone operator such that zer $A \neq \emptyset$. Let $\gamma:$ $\left[t_{0},+\infty\left[\rightarrow \mathbb{R}_{+}\right.\right.$and $\lambda:\left[t_{0},+\infty\left[\rightarrow \mathbb{R}_{+}^{*}\right.\right.$ be differentiable functions. Assuming $\left(H_{0}\right)$, let $\Gamma:\left[t_{0},+\infty\left[\rightarrow \mathbb{R}_{+}\right.\right.$ be the function defined by $\Gamma(s)=\left(\int_{s}^{+\infty} \frac{d u}{p(u)}\right) p(s)$. Suppose that there exists $\left.\varepsilon \in\right] 0,1[$ such that for $t$ large enough,

$$
(1-\varepsilon) \lambda(t) \gamma(t) \geq\left(1+\left|\frac{d}{d t}(\lambda(t) \gamma(t))\right|\right) \Gamma(t) .
$$

Then for any global solution $x($.$) of (RIMS) { }_{\gamma, \lambda}$, we have

(i) $\int_{t_{0}}^{+\infty} \lambda(s) \gamma(s)\|\dot{x}(s)\|^{2} d s<+\infty$, and as a consequence $\int_{t_{0}}^{+\infty} \Gamma(s)\|\dot{x}(s)\|^{2} d s<+\infty$.

(ii) $\int_{t_{0}}^{+\infty} \lambda(s) \Gamma(s)\left\|A_{\lambda(s)}(x(s))\right\|^{2} d s<+\infty$.

(iii) For any $z \in \operatorname{zer} A, \lim _{t \rightarrow+\infty}\|x(t)-z\|$ exists, and hence $x(\cdot)$ is bounded.

(iv) There exists a positive constant $C$ such that for $t$ large enough,

$$
\|\dot{x}(t)\| \leq \frac{C}{p(t)} \int_{t_{0}}^{t} \frac{p(s)}{\lambda(s)} d s \quad \text { and } \quad\|\ddot{x}(t)\| \leq C \frac{\gamma(t)}{p(t)} \int_{t_{0}}^{t} \frac{p(s)}{\lambda(s)} d s+\frac{C}{\lambda(t)} .
$$

Assuming that

$$
\begin{gathered}
\frac{\lambda(t)}{p(t)} \int_{t_{0}}^{t} \frac{p(s)}{\lambda(s)} d s=\mathcal{O}(\Gamma(t)) \quad \text { and } \quad|\dot{\lambda}(t)|=\mathcal{O}(\Gamma(t)) \quad \text { as } t \rightarrow+\infty, \\
\int_{t_{0}}^{+\infty} \frac{\Gamma(s)}{\lambda(s)} d s=+\infty,
\end{gathered}
$$

the following holds

(v) $\lim _{t \rightarrow+\infty} \lambda(t) A_{\lambda(t)}(x(t))=0$.

(vi) If $\lambda(\cdot)$ is minorized by some positive constant on $\left[t_{0},+\infty\left[\right.\right.$, then there exists $x_{\infty} \in$ zer $A$ such that $x(t) \rightarrow x_{\infty}$ weakly in $\mathcal{H}$ as $t \rightarrow+\infty$.

Finally assume that $\left(H_{3}\right)$ is not satisfied, i.e. $\int_{t_{0}}^{+\infty} \frac{\Gamma(s)}{\lambda(s)} d s<+\infty$. Then we obtain

(vii) $\int_{t_{0}}^{+\infty}\|\dot{x}(s)\| d s<+\infty$, and hence $x(\cdot)$ converges strongly toward some $x_{\infty} \in \mathcal{H}$.

Proof. (i) Let $z \in \operatorname{zer} A$, and let us set $h(t)=\frac{1}{2}\|x(t)-z\|^{2}$ for every $t \geq t_{0}$. By differentiating, we find for every $t \geq t_{0}$,

$$
\dot{h}(t)=\langle\dot{x}(t), x(t)-z\rangle \quad \text { and } \quad \ddot{h}(t)=\|\dot{x}(t)\|^{2}+\langle\ddot{x}(t), x(t)-z\rangle .
$$

It ensues that

$$
\begin{aligned}
\ddot{h}(t)+\gamma(t) \dot{h}(t) & =\|\dot{x}(t)\|^{2}+\langle\ddot{x}(t)+\gamma(t) \dot{x}(t), x(t)-z\rangle \\
& =\|\dot{x}(t)\|^{2}-\left\langle A_{\lambda(t)}(x(t)), x(t)-z\right\rangle
\end{aligned}
$$


Since $z \in \operatorname{zer} A=\operatorname{zer} A_{\lambda(t)}$, we have $A_{\lambda(t)}(z)=0$. We then deduce from the $\lambda(t)$-cocoercivity of $A_{\lambda(t)}$ that

$$
\left\langle A_{\lambda(t)}(x(t)), x(t)-z\right\rangle \geq \lambda(t)\left\|A_{\lambda(t)}(x(t))\right\|^{2}
$$

whence

$$
\ddot{h}(t)+\gamma(t) \dot{h}(t) \leq\|\dot{x}(t)\|^{2}-\lambda(t)\left\|A_{\lambda(t)}(x(t))\right\|^{2} .
$$

Writing that $A_{\lambda(t)}(x(t))=-\ddot{x}(t)-\gamma(t) \dot{x}(t)$, we have

$$
\begin{aligned}
\lambda(t)\left\|A_{\lambda(t)}(x(t))\right\|^{2} & =\lambda(t)\|\ddot{x}(t)+\gamma(t) \dot{x}(t)\|^{2} \\
& =\lambda(t)\|\ddot{x}(t)\|^{2}+\lambda(t) \gamma(t)^{2}\|\dot{x}(t)\|^{2}+2 \lambda(t) \gamma(t)\langle\ddot{x}(t), \dot{x}(t)\rangle \\
& \geq \lambda(t) \gamma(t)^{2}\|\dot{x}(t)\|^{2}+\lambda(t) \gamma(t) \frac{d}{d t}\|\dot{x}(t)\|^{2} \\
& =\left(\lambda(t) \gamma(t)^{2}-\frac{d}{d t}(\lambda(t) \gamma(t))\right)\|\dot{x}(t)\|^{2}+\frac{d}{d t}\left(\lambda(t) \gamma(t)\|\dot{x}(t)\|^{2}\right) .
\end{aligned}
$$

In view of (5), we infer that

$$
\ddot{h}(t)+\gamma(t) \dot{h}(t) \leq-\left(\lambda(t) \gamma(t)^{2}-\frac{d}{d t}(\lambda(t) \gamma(t))-1\right)\|\dot{x}(t)\|^{2}-\frac{d}{d t}\left(\lambda(t) \gamma(t)\|\dot{x}(t)\|^{2}\right) .
$$

Let's use Lemma B.1 $(i)$ with $g(t)=-\left(\lambda(t) \gamma(t)^{2}-\frac{d}{d t}(\lambda(t) \gamma(t))-1\right)\|\dot{x}(t)\|^{2}-\frac{d}{d t}\left(\lambda(t) \gamma(t)\|\dot{x}(t)\|^{2}\right)$. Setting $k(t):=h\left(t_{0}\right)+\dot{h}\left(t_{0}\right)\left(\int_{t_{0}}^{t} \frac{d u}{p(u)}\right)$, we obtain for every $t \geq t_{0}$,

$$
\begin{aligned}
h(t) \leq & k(t)-\int_{t_{0}}^{t} \Gamma(s, t)\left[\left(\lambda(s) \gamma(s)^{2}-\frac{d}{d s}(\lambda(s) \gamma(s))-1\right)\|\dot{x}(s)\|^{2}+\frac{d}{d s}\left(\lambda(s) \gamma(s)\|\dot{x}(s)\|^{2}\right)\right] d s \\
= & k(t)-\int_{t_{0}}^{t} \Gamma(s, t)\left(\lambda(s) \gamma(s)^{2}-\frac{d}{d s}(\lambda(s) \gamma(s))-1\right)\|\dot{x}(s)\|^{2} d s \\
& -\left[\Gamma(s, t) \lambda(s) \gamma(s)\|\dot{x}(s)\|^{2}\right]_{t_{0}}^{t}+\int_{t_{0}}^{t}\left(\frac{d}{d s} \Gamma(s, t)\right) \lambda(s) \gamma(s)\|\dot{x}(s)\|^{2} d s .
\end{aligned}
$$

Let us observe that $\Gamma(t, t)=0$ and that

$$
\frac{d}{d s} \Gamma(s, t)=\frac{d}{d s}\left[\left(\int_{s}^{t} \frac{d u}{p(u)}\right) p(s)\right]=-1+\gamma(s) \Gamma(s, t) .
$$

Then it follows from the above inequality that

$$
\begin{aligned}
h(t) \leq & k(t)-\int_{t_{0}}^{t}\left[\lambda(s) \gamma(s)-\Gamma(s, t)\left(1+\frac{d}{d s}(\lambda(s) \gamma(s))\right)\right]\|\dot{x}(s)\|^{2} d s \\
& +\Gamma\left(t_{0}, t\right) \lambda\left(t_{0}\right) \gamma\left(t_{0}\right)\left\|\dot{x}\left(t_{0}\right)\right\|^{2} .
\end{aligned}
$$

Since $\Gamma\left(t_{0}, t\right) \leq \Gamma\left(t_{0}\right)$ and $h(t) \geq 0$, we deduce that

$$
\int_{t_{0}}^{t}\left[\lambda(s) \gamma(s)-\Gamma(s, t)\left(1+\frac{d}{d s}(\lambda(s) \gamma(s))\right)\right]\|\dot{x}(s)\|^{2} d s \leq C_{1}
$$

with

Now observe that

$$
C_{1}:=h\left(t_{0}\right)+\left|\dot{h}\left(t_{0}\right)\right|\left(\int_{t_{0}}^{+\infty} \frac{d u}{p(u)}\right)+\Gamma\left(t_{0}\right) \lambda\left(t_{0}\right) \gamma\left(t_{0}\right)\left\|\dot{x}\left(t_{0}\right)\right\|^{2}
$$

$$
\Gamma(s, t)\left(1+\frac{d}{d s}(\lambda(s) \gamma(s))\right) \leq \Gamma(s, t)\left(1+\left|\frac{d}{d s}(\lambda(s) \gamma(s))\right|\right) \leq \Gamma(s)\left(1+\left|\frac{d}{d s}(\lambda(s) \gamma(s))\right|\right) .
$$


We then infer from (6) that

$$
\int_{t_{0}}^{t}\left[\lambda(s) \gamma(s)-\Gamma(s)\left(1+\left|\frac{d}{d s}(\lambda(s) \gamma(s))\right|\right)\right]\|\dot{x}(s)\|^{2} d s \leq C_{1} .
$$

By assumption, inequality $\left(H_{1}\right)$ holds true for $t$ large enough, say $t \geq t_{1}$. It ensues that for $t \geq t_{1}$,

$$
\int_{t_{1}}^{t} \varepsilon \lambda(s) \gamma(s)\|\dot{x}(s)\|^{2} d s \leq C_{1}-C_{2}
$$

with

$$
C_{2}=\int_{t_{0}}^{t_{1}}\left[\lambda(s) \gamma(s)-\Gamma(s)\left(1+\left|\frac{d}{d s}(\lambda(s) \gamma(s))\right|\right)\right]\|\dot{x}(s)\|^{2} d s .
$$

Taking the limit as $t \rightarrow+\infty$, we find

$$
\int_{t_{1}}^{+\infty} \lambda(s) \gamma(s)\|\dot{x}(s)\|^{2} d s \leq \frac{1}{\varepsilon}\left(C_{1}-C_{2}\right)<+\infty .
$$

By using again $\left(H_{1}\right)$, we deduce that $\int_{t_{1}}^{+\infty} \Gamma(s)\|\dot{x}(s)\|^{2} d s<+\infty$.

(ii) Let us come back to inequality (5). Using Lemma B.1 $(i)$ with $g(t)=\|\dot{x}(t)\|^{2}-\lambda(t)\left\|A_{\lambda(t)}(x(t))\right\|^{2}$, we obtain for every $t \geq t_{0}$,

$$
h(t) \leq h\left(t_{0}\right)+\dot{h}\left(t_{0}\right)\left(\int_{t_{0}}^{t} \frac{d u}{p(u)}\right)+\int_{t_{0}}^{t} \Gamma(s, t)\left[\|\dot{x}(s)\|^{2}-\lambda(s)\left\|A_{\lambda(s)}(x(s))\right\|^{2}\right] d s .
$$

Since $h(t) \geq 0$ and $\Gamma(s, t) \leq \Gamma(s)$, we deduce that

$$
\int_{t_{0}}^{t} \Gamma(s, t) \lambda(s)\left\|A_{\lambda(s)}(x(s))\right\|^{2} d s \leq h\left(t_{0}\right)+\dot{h}\left(t_{0}\right)\left(\int_{t_{0}}^{t} \frac{d u}{p(u)}\right)+\int_{t_{0}}^{t} \Gamma(s)\|\dot{x}(s)\|^{2} d s .
$$

Recalling from $(i)$ that $\int_{t_{0}}^{+\infty} \Gamma(s)\|\dot{x}(s)\|^{2} d s<+\infty$, we infer that for every $t \geq t_{0}$,

$$
\int_{t_{0}}^{t} \Gamma(s, t) \lambda(s)\left\|A_{\lambda(s)}(x(s))\right\|^{2} d s \leq C_{3},
$$

where we have set

$$
C_{3}:=h\left(t_{0}\right)+\left|\dot{h}\left(t_{0}\right)\right|\left(\int_{t_{0}}^{+\infty} \frac{d u}{p(u)}\right)+\int_{t_{0}}^{+\infty} \Gamma(s)\|\dot{x}(s)\|^{2} d s .
$$

Since $\Gamma(s, t)=0$ for $s \geq t$, this yields in turn

$$
\int_{t_{0}}^{+\infty} \Gamma(s, t) \lambda(s)\left\|A_{\lambda(s)}(x(s))\right\|^{2} d s \leq C_{3} .
$$

Letting $t$ tend to $+\infty$, the monotone convergence theorem then implies that

$$
\int_{t_{0}}^{+\infty} \Gamma(s) \lambda(s)\left\|A_{\lambda(s)}(x(s))\right\|^{2} d s \leq C_{3}<+\infty .
$$

(iii) From inequality (5), we derive that

$$
\ddot{h}(t)+\gamma(t) \dot{h}(t) \leq\|\dot{x}(t)\|^{2} \quad \text { on }\left[t_{0},+\infty[.\right.
$$

Recall from $(i)$ that $\int_{t_{0}}^{+\infty} \Gamma(s)\|\dot{x}(s)\|^{2} d s<+\infty$. Applying Lemma B.1 $(i i)$ with $g(t)=\|\dot{x}(t)\|^{2}$, we infer that $\lim _{t \rightarrow+\infty} h(t)$ exists. Thus, we have obtained that $\lim _{t \rightarrow+\infty}\|x(t)-z\|$ exists for every $z \in$ zer $A$, whence in particular the boundedness of the trajectory $x(\cdot)$. 
(iv) Using that the operator $A_{\lambda(t)}$ is $\frac{1}{\lambda(t)}$-Lipschitz continuous and that $A_{\lambda(t)}(z)=0$, we obtain that

$$
\left\|A_{\lambda(t)}(x(t))\right\| \leq \frac{1}{\lambda(t)}\|x(t)-z\| \leq \frac{C_{4}}{\lambda(t)},
$$

with $C_{4}:=\sup _{t \geq t_{0}}\|x(t)-z\|<+\infty$. Let us multiply (RIMS) $)_{\gamma, \lambda}$ by $p(t)=e^{\int_{t_{0}}^{t} \gamma(\tau) d \tau}$, and integrate on $\left[t_{0}, t\right]$. We find for every $t \geq t_{0}$,

$$
\dot{x}(t)=\frac{1}{p(t)} \dot{x}\left(t_{0}\right)-\frac{1}{p(t)} \int_{t_{0}}^{t} p(s) A_{\lambda(s)}(x(s)) d s .
$$

Taking the norm of each member, we deduce that

$$
\|\dot{x}(t)\| \leq \frac{1}{p(t)}\left\|\dot{x}\left(t_{0}\right)\right\|+\frac{C_{4}}{p(t)} \int_{t_{0}}^{t} \frac{p(s)}{\lambda(s)} d s .
$$

Hence there exists $C_{5} \geq C_{4}$ such that for $t$ large enough

$$
\|\dot{x}(t)\| \leq \frac{C_{5}}{p(t)} \int_{t_{0}}^{t} \frac{p(s)}{\lambda(s)} d s .
$$

This proves the first inequality of $(i v)$. For the second one, take the norm of each member of the equality $\ddot{x}(t)=-\gamma(t) \dot{x}(t)-A_{\lambda(t)}(x(t))$. The triangle inequality yields

$$
\|\ddot{x}(t)\| \leq \gamma(t)\|\dot{x}(t)\|+\left\|A_{\lambda(t)}(x(t))\right\| .
$$

The announced majorization of $\|\ddot{x}(t)\|$ then follows from (7) and (8).

$(v)$ Recall the estimate of $(i i)$ that we write as

$$
\int_{t_{0}}^{+\infty} \frac{\Gamma(s)}{\lambda(s)}\|u(s)\|^{2} d s<+\infty
$$

with the function $u:\left[t_{0},+\infty\left[\rightarrow \mathcal{H}\right.\right.$ defined by $u(t)=\lambda(t) A_{\lambda(t)}(x(t))$. By applying [8, Lemma A.4] with $\gamma=\lambda(t), \delta=\lambda(s), x=x(t)$ and $y=x(s)$ with $s, t \geq t_{0}$, we find

$$
\left\|\lambda(t) A_{\lambda(t)}(x(t))-\lambda(s) A_{\lambda(s)}(x(s))\right\| \leq 2\|x(t)-x(s)\|+2\|x(t)-z\| \frac{|\lambda(t)-\lambda(s)|}{\lambda(t)} .
$$

This shows that the map $t \mapsto \lambda(t) A_{\lambda(t)}(x(t))$ is locally Lipschitz continuous, hence almost everywhere differentiable on $\left[t_{0},+\infty[\right.$. Dividing by $t-s$ with $t \neq s$, and letting $s$ tend to $t$, we infer that

$$
\|\dot{u}(t)\|=\left\|\frac{d}{d t}\left(\lambda(t) A_{\lambda(t)}(x(t))\right)\right\| \leq 2\|\dot{x}(t)\|+2\|x(t)-z\| \frac{|\dot{\lambda}(t)|}{\lambda(t)},
$$

for almost every $t \geq t_{0}$. In view of (8), we deduce that for almost every $t$ large enough,

$$
\|\dot{u}(t)\| \leq 2 \frac{C_{5}}{p(t)} \int_{t_{0}}^{t} \frac{p(s)}{\lambda(s)} d s+2 C_{4} \frac{|\dot{\lambda}(t)|}{\lambda(t)},
$$

with $C_{4}=\sup _{t>t_{0}}\|x(t)-z\|<+\infty$. Recalling the assumption $\left(H_{2}\right)$, we obtain the existence of $C_{6} \geq 0$ such that for almost every $t$ large enough

$$
\|\dot{u}(t)\| \leq C_{6} \frac{\Gamma(t)}{\lambda(t)} .
$$

Then we have

$$
\frac{d}{d t}\|u(t)\|^{3} \leq 3\|\dot{u}(t)\|\|u(t)\|^{2} \leq 3 C_{6} \frac{\Gamma(t)}{\lambda(t)}\|u(t)\|^{2} .
$$


Taking account of estimate (9), this shows that

$$
\left(\frac{d}{d t}\|u(t)\|^{3}\right)_{+} \in L^{1}\left(t_{0},+\infty\right)
$$

From a classical result, this implies that $\lim _{t \rightarrow+\infty}\|u(t)\|^{3}$ exists, which entails in turn that $\lim _{t \rightarrow+\infty}\|u(t)\|$ exists. Using again the estimate (9), together with the assumption $\left(H_{3}\right)$, we immediately conclude that $\lim _{t \rightarrow+\infty}\|u(t)\|=0$.

$(v i)$ To prove the weak convergence of $x(t)$ as $t \rightarrow+\infty$, we use the Opial lemma with $S=$ zer $A$. Item $(i i i)$ shows the first condition of the Opial lemma. For the second one, let $t_{n} \rightarrow+\infty$ be such that $x\left(t_{n}\right) \rightarrow \bar{x}$ weakly as $n \rightarrow+\infty$. By $(v)$, we have $\lim _{n \rightarrow+\infty} \lambda\left(t_{n}\right) A_{\lambda\left(t_{n}\right)}\left(x\left(t_{n}\right)\right)=0$ strongly in $\mathcal{H}$. Since the function $\lambda$ is minorized by some positive constant on $\left[t_{0},+\infty\right.$ [, we also have $\lim _{n \rightarrow+\infty} A_{\lambda\left(t_{n}\right)}\left(x\left(t_{n}\right)\right)=0$ strongly in $\mathcal{H}$. Passing to the limit in

$$
A_{\lambda\left(t_{n}\right)}\left(x\left(t_{n}\right)\right) \in A\left(x\left(t_{n}\right)-\lambda\left(t_{n}\right) A_{\lambda\left(t_{n}\right)}\left(x\left(t_{n}\right)\right)\right),
$$

and invoking the graph-closedness of the maximally monotone operator $A$ for the weak-strong topology in $\mathcal{H} \times \mathcal{H}$, we find $0 \in A(\bar{x})$. This shows that $\bar{x} \in \operatorname{zer} A$, which completes the proof.

(vii) Let us now assume that $\int_{t_{0}}^{+\infty} \frac{\Gamma(s)}{\lambda(s)} d s<+\infty$. Recalling inequality (7), we deduce that

$$
\int_{t_{0}}^{+\infty} \Gamma(s)\left\|A_{\lambda(s)}(x(s))\right\| d s<+\infty .
$$

By applying Lemma B.2 with $F(t)=-A_{\lambda(t)}(x(t))$, we obtain that $\int_{t_{0}}^{+\infty}\|\dot{x}(s)\| d s<+\infty$, and hence $x(t)$ converges strongly as $t \rightarrow+\infty$ toward some $x_{\infty} \in \mathcal{H}$.

Remark 2.4. When $\int_{t_{0}}^{+\infty} \frac{\Gamma(s)}{\lambda(s)} d s<+\infty$, the trajectories of (RIMS) ${ }_{\gamma, \lambda}$ have a finite length, and hence are strongly convergent. However, the limit point is not a zero of the operator $A$ in general.

Let us now particularize Theorem 2.3 to the case of a constant parameter $\lambda>0$. In this case, the operator arising in equation (RIMS) ${ }_{\gamma, \lambda}$ is constant and equal to the $\lambda$-cocoercive operator $A_{\lambda}$. On the other hand, it is well-known that every $\lambda$-cocoercive operator $B: \mathcal{H} \rightarrow \mathcal{H}$ can be viewed as the Yosida regularization $A_{\lambda}$ of some maximally monotone operator $A: \mathcal{H} \rightarrow 2^{\mathcal{H}}$, see [11, Proposition 23.20]. This leads to the following statement.

Corollary 2.5. Let $\lambda>0$ and let $B: \mathcal{H} \rightarrow \mathcal{H}$ be a $\lambda$-cocoercive operator such that zer $B \neq \emptyset$. Given a differentiable function $\gamma:\left[t_{0},+\infty\left[\rightarrow \mathbb{R}_{+}\right.\right.$satisfying $\left(H_{0}\right)$, let $\Gamma, \Delta:\left[t_{0},+\infty\left[\rightarrow \mathbb{R}_{+}\right.\right.$be the functions respectively defined by $\Gamma(s)=p(s)\left(\int_{s}^{+\infty} \frac{d u}{p(u)}\right)$ and $\Delta(s)=\frac{1}{p(s)}\left(\int_{t_{0}}^{s} p(u) d u\right)$. Assume that there exists $\varepsilon \in] 0,1[$ such that for s large enough,

$$
(1-\varepsilon) \lambda \gamma(s) \geq(1+\lambda|\dot{\gamma}(s)|) \Gamma(s) .
$$

Then for any global solution $x($.$) of$

$$
\ddot{x}(t)+\gamma(t) \dot{x}(t)+B(x(t))=0, \quad t \geq t_{0},
$$

we have

(i) $\int_{t_{0}}^{+\infty} \gamma(s)\|\dot{x}(s)\|^{2} d s<+\infty$, and as a consequence $\int_{t_{0}}^{+\infty} \Gamma(s)\|\dot{x}(s)\|^{2} d s<+\infty$.

(ii) $\int_{t_{0}}^{+\infty} \Gamma(s)\|B(x(s))\|^{2} d s<+\infty$.

(iii) For any $z \in \operatorname{zer} B, \lim _{t \rightarrow+\infty}\|x(t)-z\|$ exists, and hence $x(\cdot)$ is bounded.

(iv) There exists $C \geq 0$ such that for $t$ large enough,

$$
\|\dot{x}(t)\| \leq C \Delta(t) \quad \text { and } \quad\|\ddot{x}(t)\| \leq C \gamma(t) \Delta(t)+C .
$$


Assuming that $\int_{t_{0}}^{+\infty} \Gamma(s) d s=+\infty$, and that $\Delta(t)=\mathcal{O}(\Gamma(t))$ as $t \rightarrow+\infty$, the following holds

(v) $\lim _{t \rightarrow+\infty} B(x(t))=0$.

(vi) There exists $x_{\infty} \in \operatorname{zer} B$ such that $x(t) \rightarrow x_{\infty}$ weakly in $\mathcal{H}$ as $t \rightarrow+\infty$.

Finally assume that $\int_{t_{0}}^{+\infty} \Gamma(s) d s<+\infty$. Then we obtain

(vii) $\int_{t_{0}}^{+\infty}\|\dot{x}(s)\| d s<+\infty$, and hence $x(\cdot)$ converges strongly toward some $x_{\infty} \in \mathcal{H}$.

Assume now that the function $\gamma$ is constant, say $\gamma(t) \equiv \gamma>0$. In this case, it is easy to check that

$$
\Gamma(t) \sim \frac{1}{\gamma} \quad \text { and } \quad \Delta(t) \sim \frac{1}{\gamma} \quad \text { as } t \rightarrow+\infty
$$

see Proposition 3.1. As a consequence of Corollary 2.5, we then obtain the following result that was originally discovered by Attouch-Maingé [6].

Corollary 2.6 (Attouch-Maingé [6]). Let $\lambda>0$ and let $B: \mathcal{H} \rightarrow \mathcal{H}$ be a $\lambda$-cocoercive operator such that $\operatorname{zer} B \neq \emptyset$. Let $\gamma>0$ be such that $\lambda \gamma^{2}>1$. Then for any global solution $x($.$) of$

$$
\ddot{x}(t)+\gamma \dot{x}(t)+B(x(t))=0, \quad t \geq t_{0},
$$

we have

(i) $\int_{t_{0}}^{+\infty}\|\dot{x}(s)\|^{2} d s<+\infty$.

(ii) $\int_{t_{0}}^{+\infty}\|B(x(s))\|^{2} d s<+\infty$.

(iii) For any $z \in \operatorname{zer} B, \lim _{t \rightarrow+\infty}\|x(t)-z\|$ exists, and hence $x(\cdot)$ is bounded.

(iv) $\lim _{t \rightarrow+\infty} \dot{x}(t)=0$ and $\lim _{t \rightarrow+\infty} \ddot{x}(t)=0$.

(v) $\lim _{t \rightarrow+\infty} B(x(t))=0$.

(vi) There exists $x_{\infty} \in \operatorname{zer} B$ such that $x(t) \rightarrow x_{\infty}$ weakly in $\mathcal{H}$ as $t \rightarrow+\infty$.

Proof. Since $\gamma(t) \equiv \gamma>0$, we have the equivalences (11) as $t \rightarrow+\infty$. It ensues that condition (10) is guaranteed by $\lambda \gamma^{2}>1$. All points are then obvious consequences of Corollary 2.5, except for (iv). Corollary $2.5(i v)$ shows that the acceleration $\ddot{x}$ is bounded on $\left[t_{0},+\infty[\right.$. Taking account of $(i)$, we deduce classically that $\lim _{t \rightarrow+\infty} \dot{x}(t)=0$. In view of equation (12) and the fact that $\lim _{t \rightarrow+\infty} B(x(t))=0$ by $(v)$, we conclude that $\lim _{t \rightarrow+\infty} \ddot{x}(t)=0$.

\section{ApplicAtion to PARTiCUlar CLASSES OF FUNCTIONS $\gamma$ AND $\lambda$}

We now look at special classes of functions $\gamma$ and $\lambda$, for which we are able to estimate precisely the quantities $\int_{t}^{+\infty} \frac{d s}{p(s)}$ and $\int_{t_{0}}^{t} \frac{p(s)}{\lambda(s)} d s$ as $t \rightarrow+\infty$. This consists of the differentiable functions $\gamma$, $\lambda:\left[t_{0},+\infty\left[\rightarrow \mathbb{R}_{+}^{*}\right.\right.$ satisfying

$$
\lim _{t \rightarrow+\infty} \frac{\dot{\gamma}(t)}{\gamma(t)^{2}}=-c \quad \text { and } \quad \lim _{t \rightarrow+\infty} \frac{\frac{d}{d t}(\lambda(t) \gamma(t))}{\lambda(t) \gamma(t)^{2}}=-c^{\prime},
$$

for some $c \in\left[0,1\left[\right.\right.$ and $c^{\prime}>-1$. Some properties of the functions $\gamma$ satisfying the first condition above were studied by Attouch-Cabot [3], in connection with the asymptotic behavior of the inertial gradient system (IGS) $)_{\gamma}$. The next proposition extends some of these properties.

Proposition 3.1. Let $\gamma, \lambda:\left[t_{0},+\infty\left[\rightarrow \mathbb{R}_{+}^{*}\right.\right.$ be two differentiable functions. 
(i) Assume that there exists $c \in\left[0,1\left[\right.\right.$ such that $\lim _{t \rightarrow+\infty} \dot{\gamma}(t) / \gamma(t)^{2}=-c$. Then the conditions $\int_{t_{0}}^{+\infty} \gamma(s) d s=+\infty$ and $\left(H_{0}\right)$ are satisfied, and

$$
\int_{t}^{+\infty} \frac{d s}{p(s)} \sim \frac{1}{(1-c) p(t) \gamma(t)} \quad \text { as } t \rightarrow+\infty
$$

The above equivalence can be reformulated as $\Gamma(t) \sim \frac{1}{(1-c) \gamma(t)}$ as $t \rightarrow+\infty$.

(ii) Assume moreover that there exists $c^{\prime}>-1$ such that

$$
\lim _{t \rightarrow+\infty} \frac{\frac{d}{d t}(\lambda(t) \gamma(t))}{\lambda(t) \gamma(t)^{2}}=-c^{\prime}
$$

Then we have $\int_{t_{0}}^{+\infty} \frac{p(s)}{\lambda(s)} d s=+\infty$ and the following equivalence holds true

$$
\int_{t_{0}}^{t} \frac{p(s)}{\lambda(s)} d s \sim \frac{1}{1+c^{\prime}} \frac{p(t)}{\lambda(t) \gamma(t)} \quad \text { as } t \rightarrow+\infty .
$$

Proof. (i) This result was proved by the authors in a previous paper, see [3, Proposition 2.6].

(ii) Let us evaluate the derivative of the function $t \mapsto \frac{p(t)}{\lambda(t) \gamma(t)}$

$$
\frac{d}{d t}\left(\frac{p(t)}{\lambda(t) \gamma(t)}\right)=-\frac{\frac{d}{d t}(\lambda(t) \gamma(t))}{\lambda(t)^{2} \gamma(t)^{2}} p(t)+\frac{1}{\lambda(t) \gamma(t)} \gamma(t) p(t)=\left(-\frac{\frac{d}{d t}(\lambda(t) \gamma(t))}{\lambda(t) \gamma(t)^{2}}+1\right) \frac{p(t)}{\lambda(t)} .
$$

Since $\lim _{t \rightarrow+\infty} \frac{\frac{d}{d t}(\lambda(t) \gamma(t))}{\lambda(t) \gamma(t)^{2}}=-c^{\prime}$, we infer from the above equality that

$$
\frac{d}{d t}\left(\frac{p(t)}{\lambda(t) \gamma(t)}\right) \sim\left(1+c^{\prime}\right) \frac{p(t)}{\lambda(t)} \quad \text { as } t \rightarrow+\infty .
$$

Recalling that $c^{\prime}>-1$, we deduce that the function $t \mapsto \frac{p(t)}{\lambda(t) \gamma(t)}$ is increasing for $t$ large enough. This implies that this function is minorized on $\left[t_{0},+\infty\right.$ [ by some $m>0$. Writing that

$$
\frac{p(t)}{\lambda(t)}=\frac{p(t)}{\lambda(t) \gamma(t)} \gamma(t) \geq m \gamma(t)
$$

and using that $\int_{t_{0}}^{+\infty} \gamma(s) d s=+\infty$ by $(i)$, we conclude that $\int_{t_{0}}^{+\infty} \frac{p(s)}{\lambda(s)} d s=+\infty$. Integrating the equivalence (14), we then obtain

$$
\frac{p(t)}{\lambda(t) \gamma(t)} \sim\left(1+c^{\prime}\right) \int_{t_{0}}^{t} \frac{p(s)}{\lambda(s)} d s \quad \text { as } t \rightarrow+\infty,
$$

which completes the proof.

We now show that the key condition $\left(H_{1}\right)$ of Theorem 2.3 takes a simple form for functions $\gamma$ and $\lambda$ satisfying conditions (13).

Proposition 3.2. Let $\gamma, \lambda:\left[t_{0},+\infty\left[\rightarrow \mathbb{R}_{+}^{*}\right.\right.$ be two differentiable functions satisfying conditions (13) for some $c \in\left[0,1\left[\right.\right.$ and $\left.c^{\prime} \in\right]-1,1\left[\right.$ such that $\left|c^{\prime}\right|<1-c$. Then condition $\left(H_{1}\right)$ is equivalent to

$$
\liminf _{t \rightarrow+\infty} \lambda(t) \gamma(t)^{2}>\frac{1}{1-c-\left|c^{\prime}\right|} .
$$

Proof. The inequality arising in condition $\left(H_{1}\right)$ can be rewritten as

$$
(1-\varepsilon) \lambda(t) \frac{\gamma(t)}{\Gamma(t)}-\left|\frac{d}{d t}(\lambda(t) \gamma(t))\right| \geq 1 .
$$


The assumption $\lim _{t \rightarrow+\infty} \frac{\dot{\gamma}(t)}{\gamma(t)^{2}}=-c$ implies that $\Gamma(t) \sim \frac{1}{(1-c) \gamma(t)}$ as $t \rightarrow+\infty$, see Proposition $3.1(i)$. It ensues that

$$
\lambda(t) \frac{\gamma(t)}{\Gamma(t)}=(1-c) \lambda(t) \gamma(t)^{2}+o\left(\lambda(t) \gamma(t)^{2}\right) \quad \text { as } t \rightarrow+\infty .
$$

On the other hand, we deduce from the second condition of (13) that

$$
\left|\frac{d}{d t}(\lambda(t) \gamma(t))\right|=\left|c^{\prime}\right| \lambda(t) \gamma(t)^{2}+o\left(\lambda(t) \gamma(t)^{2}\right) \quad \text { as } t \rightarrow+\infty .
$$

In view of (17) and (18), inequality (16) amounts to

$$
\lambda(t) \gamma(t)^{2}\left[(1-\varepsilon)(1-c)-\left|c^{\prime}\right|+o(1)\right] \geq 1 \quad \text { as } t \rightarrow+\infty .
$$

Therefore condition $\left(H_{1}\right)$ is equivalent to the existence of $\left.\varepsilon^{\prime} \in\right] 0,1-c-\left|c^{\prime}\right|[$ such that

$$
\lambda(t) \gamma(t)^{2}\left[1-c-\left|c^{\prime}\right|-\varepsilon^{\prime}\right] \geq 1,
$$

for $t$ large enough. This last condition is equivalent to (15), which ends the proof.

Combining Theorem 2.3 and Propositions 3.1 and 3.2, we obtain the following result.

Corollary 3.3. Let $A: \mathcal{H} \rightarrow 2^{\mathcal{H}}$ be a maximally monotone operator such that $\operatorname{zer} A \neq \emptyset$. Let $\gamma$, $\lambda:\left[t_{0},+\infty\left[\rightarrow \mathbb{R}_{+}^{*}\right.\right.$ be two differentiable functions satisfying conditions (13) for some $c \in[0,1[$ and $\left.c^{\prime} \in\right]-1,1\left[\right.$ such that $\left|c^{\prime}\right|<1-c$. Assume moreover that

$$
\liminf _{t \rightarrow+\infty} \lambda(t) \gamma(t)^{2}>\frac{1}{1-c-\left|c^{\prime}\right|} .
$$

Then for any global solution $x($.$) of (RIMS )_{\gamma, \lambda}$, we have

(i) $\int_{t_{0}}^{+\infty} \lambda(s) \gamma(s)\|\dot{x}(s)\|^{2} d s<+\infty$.

(ii) $\int_{t_{0}}^{+\infty} \frac{\lambda(s)}{\gamma(s)}\left\|A_{\lambda(s)}(x(s))\right\|^{2} d s<+\infty$.

(iii) For any $z \in \operatorname{zer} A, \lim _{t \rightarrow+\infty}\|x(t)-z\|$ exists, and hence $x(\cdot)$ is bounded.

(iv) $\|\dot{x}(t)\|=\mathcal{O}\left(\frac{1}{\lambda(t) \gamma(t)}\right)$ and $\|\ddot{x}(t)\|=\mathcal{O}\left(\frac{1}{\lambda(t)}\right)$ as $t \rightarrow+\infty$.

Assuming that $\int_{t_{0}}^{+\infty} \frac{1}{\lambda(s) \gamma(s)} d s=+\infty$ and that $|\dot{\lambda}(t)|=\mathcal{O}\left(\frac{1}{\gamma(t)}\right)$ as $t \rightarrow+\infty$, the following holds

(v) $\lim _{t \rightarrow+\infty} \lambda(t) A_{\lambda(t)}(x(t))=0$.

(vi) If $\lambda$ is minorized by some positive constant on $\left[t_{0},+\infty\left[\right.\right.$, there exists $x_{\infty} \in$ zer $A$ such that $x(t) \rightarrow$ $x_{\infty}$ weakly in $\mathcal{H}$ as $t \rightarrow+\infty$.

Finally assume that $\int_{t_{0}}^{+\infty} \frac{1}{\lambda(s) \gamma(s)} d s<+\infty$. Then we obtain

(vii) $\int_{t_{0}}^{+\infty}\|\dot{x}(s)\| d s<+\infty$, and hence $x(\cdot)$ converges strongly toward some $x_{\infty} \in \mathcal{H}$.

Proof. The key condition $\left(H_{1}\right)$ of Theorem 2.3 is satisfied owing to Proposition 3.2. On the other hand, Proposition 3.1 shows that

$$
\Gamma(t) \sim \frac{1}{(1-c) \gamma(t)} \quad \text { and } \quad \frac{1}{p(t)} \int_{t_{0}}^{t} \frac{p(s)}{\lambda(s)} d s \sim \frac{1}{\left(1+c^{\prime}\right) \lambda(t) \gamma(t)} \quad \text { as } t \rightarrow+\infty .
$$


It ensues that the first condition of $\left(H_{2}\right)$ is automatically satisfied, while the second one is given by $|\dot{\lambda}(t)|=\mathcal{O}\left(\frac{1}{\gamma(t)}\right)$ as $t \rightarrow+\infty$. Condition $\left(H_{3}\right)$ is implied by the assumption $\int_{t_{0}}^{+\infty} \frac{1}{\lambda(s) \gamma(s)} d s=+\infty$. Items $(i)$-(vii) follow immediately from the corresponding points in Theorem 2.3.

Let us now particularize to the case $\gamma(t)=\alpha t^{q}$ and $\lambda(t)=\beta t^{r}$, for some $\alpha, \beta>0, q \geq-1$ and $r \in \mathbb{R}$.

Corollary 3.4. Let $A: \mathcal{H} \rightarrow 2^{\mathcal{H}}$ be a maximally monotone operator such that $\operatorname{zer} A \neq \emptyset$. Assume that $\gamma(t)=\alpha t^{q}$ and $\lambda(t)=\beta t^{r}$ for every $t \geq t_{0}>0$. Suppose that $\left.(q, r) \in\right]-1,+\infty[\times \mathbb{R}$ is such that $2 q+r \geq 0$, and that $(\alpha, \beta) \in \mathbb{R}_{+}^{*} \times \mathbb{R}_{+}^{*}$ satisfies $\alpha^{2} \beta>1$ if $2 q+r=0$ (no condition if $2 q+r>0$ ). Then for any global solution $x($.$) of (RIMS )_{\gamma, \lambda}$, we have

(i) $\int_{t_{0}}^{+\infty} s^{q+r}\|\dot{x}(s)\|^{2} d s<+\infty$.

(ii) $\int_{t_{0}}^{+\infty} s^{r-q}\left\|A_{\lambda(s)}(x(s))\right\|^{2} d s<+\infty$.

(iii) For any $z \in \operatorname{zer} A, \lim _{t \rightarrow+\infty}\|x(t)-z\|$ exists, and hence $x(\cdot)$ is bounded.

(iv) $\|\dot{x}(t)\|=\mathcal{O}\left(\frac{1}{t^{q+r}}\right)$ and $\|\ddot{x}(t)\|=\mathcal{O}\left(\frac{1}{t^{r}}\right)$ as $t \rightarrow+\infty$.

Assuming that $q+r \leq 1$, the following holds

(v) $\lim _{t \rightarrow+\infty} t^{r} A_{\lambda(t)}(x(t))=0$.

(vi) If $r \geq 0$, there exists $x_{\infty} \in \operatorname{zer} A$ such that $x(t) \rightarrow x_{\infty}$ weakly in $\mathcal{H}$ as $t \rightarrow+\infty$.

Finally assume that $q+r>1$. Then we obtain

(vii) $\int_{t_{0}}^{+\infty}\|\dot{x}(s)\| d s<+\infty$, and hence $x(\cdot)$ converges strongly toward some $x_{\infty} \in \mathcal{H}$.

Proof. Since $q>-1$, the first (resp. second) condition of (13) is satisfied with $c=0$ (resp. $\left.c^{\prime}=0\right)$. On the other hand, we have $\lambda(t) \gamma(t)^{2}=\alpha^{2} \beta t^{2 q+r}$, hence

$$
\lim _{t \rightarrow+\infty} \lambda(t) \gamma(t)^{2}=\left\{\begin{array}{lll}
+\infty & \text { if } & 2 q+r>0 \\
\alpha^{2} \beta & \text { if } & 2 q+r=0 .
\end{array}\right.
$$

It ensues that the condition $\liminf _{t \rightarrow+\infty} \lambda(t) \gamma(t)^{2}>1$ is guaranteed by the hypotheses of Corollary 3.4. Conditions $\int_{t_{0}}^{+\infty} \frac{d s}{\lambda(s) \gamma(s)}=+\infty$ and $|\dot{\lambda}(t)|=\mathcal{O}(1 / \gamma(t))$ as $t \rightarrow+\infty$ amount respectively to $q+r \leq 1$. Items $(i)-(v i i)$ are immediate consequences of the corresponding points in Corollary 3.3.

When $q=r=0$, the functions $\gamma$ and $\lambda$ are constant: $\gamma(t) \equiv \alpha>0$ and $\lambda(t) \equiv \beta>0$. We then recover the result of [6, Theorem 2.1] with the key condition $\alpha^{2} \beta>1$. To finish, let us consider the case $q=-1$, thus leading to a damping parameter of the form $\gamma(t)=\frac{\alpha}{t}$. This case was recently studied by Attouch and Peypouquet [8] in the framework of Nesterov's accelerated methods.

Corollary 3.5. Let $A: \mathcal{H} \rightarrow 2^{\mathcal{H}}$ be a maximally monotone operator such that zer $A \neq \emptyset$. Let $r \geq 2$, $\alpha>r$ and $\beta \in \mathbb{R}_{+}^{*}$ be such that $\beta>\frac{1}{\alpha(\alpha-r)}$ if $r=2$ (no condition on $\beta$ if $r>2$ ). Assume that $\gamma(t)=\frac{\alpha}{t}$ and $\lambda(t)=\beta t^{r}$ for every $t \geq t_{0}>0$. Then for any global solution $x($.$) of (RIMS) { }_{\gamma, \lambda}$, we have

(i) $\int_{t_{0}}^{+\infty} s^{r-1}\|\dot{x}(s)\|^{2} d s<+\infty$.

(ii) $\int_{t_{0}}^{+\infty} s^{r+1}\left\|A_{\lambda(s)}(x(s))\right\|^{2} d s<+\infty$.

(iii) For any $z \in \operatorname{zer} A, \lim _{t \rightarrow+\infty}\|x(t)-z\|$ exists, and hence $x(\cdot)$ is bounded.

(iv) $\|\dot{x}(t)\|=\mathcal{O}\left(\frac{1}{t^{r-1}}\right)$ and $\|\ddot{x}(t)\|=\mathcal{O}\left(\frac{1}{t^{r}}\right)$ as $t \rightarrow+\infty$. 
Assuming that $r=2$, the following holds

(v) $\lim _{t \rightarrow+\infty} t^{2} A_{\lambda(t)}(x(t))=0$.

(vi) There exists $x_{\infty} \in \operatorname{zer} A$ such that $x(t) \rightarrow x_{\infty}$ weakly in $\mathcal{H}$ as $t \rightarrow+\infty$.

Finally assume that $r>2$. Then we obtain

(vii) $\int_{t_{0}}^{+\infty}\|\dot{x}(s)\| d s<+\infty$, and hence $x(\cdot)$ converges strongly toward some $x_{\infty} \in \mathcal{H}$.

Proof. The first (resp. second) condition of (13) is satisfied with $c=\frac{1}{\alpha}$ (resp. $\left.c^{\prime}=\frac{1-r}{\alpha}\right)$. Since $r \geq 2$ and $\alpha>r$, we have

$$
c \in] 0,1 / 2\left[\quad \text { and } \quad\left|c^{\prime}\right|=\frac{r-1}{\alpha}<\frac{\alpha-1}{\alpha}=1-c .\right.
$$

On the other hand, observe that $\lambda(t) \gamma(t)^{2}=\alpha^{2} \beta t^{r-2}$, hence

$$
\lim _{t \rightarrow+\infty} \lambda(t) \gamma(t)^{2}=\left\{\begin{array}{lll}
+\infty & \text { if } & r>2 \\
\alpha^{2} \beta & \text { if } & r=2
\end{array}\right.
$$

Condition $\liminf \operatorname{int}_{t \rightarrow+\infty} \lambda(t) \gamma(t)^{2}>\frac{1}{1-c-\left|c^{\prime}\right|}$ is automatically satisfied if $r>2$, while it amounts to

$$
\alpha^{2} \beta>\frac{1}{1-\frac{1}{\alpha}-\frac{r-1}{\alpha}}=\frac{\alpha}{\alpha-r} \Longleftrightarrow \beta>\frac{1}{\alpha(\alpha-r)} \quad \text { if } r=2 .
$$

Items $(i)$-(vii) follow immediately from the corresponding points in Corollary 3.3.

Taking $r=2$ in the previous corollary, we recover the result of [8, Theorem 2.1] as a particular case.

\section{PART B: ERGODIC CONVERGENCE RESULTS}

Let $A: \mathcal{H} \rightarrow 2^{\mathcal{H}}$ be a maximally monotone operator. The trajectories associated to the semigroup of contractions generated by $A$ are known to converge weakly in average toward some zero of $A$, $c f$. the seminal paper by Brezis and Baillon [9]. Our purpose in this part of the paper is to study the ergodic convergence of the solutions of the system (RIMS) ${ }_{\gamma, \lambda}$. When the regularizing parameter $\lambda(\cdot)$ is minorized by some positive constant, it is established in part A that the trajectories of (RIMS) ${ }_{\gamma, \lambda}$ do converge weakly toward a zero of $A$, see Theorem $2.3(v i)$. Our objective is to show that weak ergodic convergence can be expected when the regularization parameter $\lambda(t)$ tends toward 0 as $t \rightarrow+\infty$. The key ingredient is the use of some suitable ergodic variant of the Opial lemma.

\section{WEAK ERGODIC CONVERGENCE OF THE TRAJECTORIES}

4.1. Ergodic variants of Opial's lemma. Ergodic versions of the Opial lemma were derived by BrézisBrowder [16] and Passty [26] in a discrete setting. In order to give a continuous ergodic version, let us consider a measurable function $\Lambda:\left[t_{0},+\infty\left[\times\left[t_{0},+\infty\left[\rightarrow \mathbb{R}_{+}\right.\right.\right.\right.$satisfying the following assumptions

$$
\begin{gathered}
\int_{t_{0}}^{+\infty} \Lambda(s, t) d s=1 \quad \text { for every } t \geq t_{0}, \\
\lim _{t \rightarrow+\infty} \int_{t_{0}}^{T} \Lambda(s, t) d s=0 \quad \text { for every } T \geq t_{0} .
\end{gathered}
$$

To each bounded map $x:\left[t_{0},+\infty\left[\rightarrow \mathcal{H}\right.\right.$, we associate the averaged map $\widehat{x}:\left[t_{0},+\infty[\rightarrow \mathcal{H}\right.$ by

$$
\widehat{x}(t)=\int_{t_{0}}^{+\infty} \Lambda(s, t) x(s) d s .
$$


Lemma B.4 in the appendix shows that the map $\widehat{x}$ is well-defined, bounded and that convergence of $x(t)$ as $t \rightarrow+\infty$ implies convergence of $\widehat{x}(t)$ toward the same limit (Cesaro property). The extension of Opial lemma to a general averaging process satisfying (19) and (20) is given hereafter. This result was established in [5] for the particular case corresponding to $\Lambda(s, t)=\frac{1}{t}$ if $s \leq t$ and $\Lambda(s, t)=0$ if $s>t$.

Proposition 4.1. Let $S$ be a nonempty subset of $\mathcal{H}$ and let $x:\left[t_{0},+\infty[\rightarrow \mathcal{H}\right.$ be a continuous map, supposed to be bounded on $\left[t_{0},+\infty\left[\right.\right.$. Let $\Lambda:\left[t_{0},+\infty\left[\times\left[t_{0},+\infty\left[\rightarrow \mathbb{R}_{+}\right.\right.\right.\right.$be a measurable function satisfying (19) and (20), and let $\widehat{x}:\left[t_{0},+\infty[\rightarrow \mathcal{H}\right.$ be the averaged trajectory defined by (21). Assume that

(i) for every $z \in S, \lim _{t \rightarrow+\infty}\|x(t)-z\|$ exists;

(ii) every weak sequential limit point of $\widehat{x}(t)$, as $t \rightarrow+\infty$, belongs to $S$.

Then $\widehat{x}(t)$ converges weakly as $t \rightarrow+\infty$ to a point in $S$.

Proof. From Lemma B.4 $(i)$, the map $\widehat{x}$ is bounded, therefore it is enough to establish the uniqueness of weak limit points. Let $\left(\widehat{x}\left(t_{n}\right)\right)$ and $\left(\widehat{x}\left(t_{m}\right)\right)$ be two weakly converging subsequences satisfying respectively $\widehat{x}\left(t_{n}\right) \rightarrow x_{1}$ as $n \rightarrow+\infty$ and $\widehat{x}\left(t_{m}\right) \rightarrow x_{2}$ as $m \rightarrow+\infty$. From $(i i)$, the weak limit points $x_{1}$ and $x_{2}$ belong to $S$. In view of $(i)$, we deduce that $\lim _{t \rightarrow+\infty}\left\|x(t)-x_{1}\right\|^{2}$ and $\lim _{t \rightarrow+\infty}\left\|x(t)-x_{2}\right\|^{2}$ exist. Writing that

$$
\left\|x(t)-x_{1}\right\|^{2}-\left\|x(t)-x_{2}\right\|^{2}=2\left\langle x(t)-\frac{x_{1}+x_{2}}{2}, x_{2}-x_{1}\right\rangle,
$$

we infer that $\lim _{t \rightarrow+\infty}\left\langle x(t), x_{2}-x_{1}\right\rangle$ exists. Observe that

$$
\begin{aligned}
\left\langle\widehat{x}(t), x_{2}-x_{1}\right\rangle & =\left\langle\int_{t_{0}}^{+\infty} \Lambda(s, t) x(s) d s, x_{2}-x_{1}\right\rangle \\
& =\int_{t_{0}}^{+\infty} \Lambda(s, t)\left\langle x(s), x_{2}-x_{1}\right\rangle d s .
\end{aligned}
$$

By applying Lemma B.4 (ii) to the real-valued map $t \mapsto\left\langle x(t), x_{2}-x_{1}\right\rangle$, we deduce that $\lim _{t \rightarrow+\infty}\left\langle\widehat{x}(t), x_{2}-\right.$ $\left.x_{1}\right\rangle$ exists. This implies that

$$
\lim _{n \rightarrow+\infty}\left\langle\widehat{x}\left(t_{n}\right), x_{2}-x_{1}\right\rangle=\lim _{m \rightarrow+\infty}\left\langle\widehat{x}\left(t_{m}\right), x_{2}-x_{1}\right\rangle,
$$

which entails that $\left\langle x_{1}, x_{2}-x_{1}\right\rangle=\left\langle x_{2}, x_{2}-x_{1}\right\rangle$. We conclude that $\left\|x_{2}-x_{1}\right\|^{2}=0$, which ends the proof.

Now assume that the function $\Lambda:\left[t_{0},+\infty\left[\times\left[t_{0},+\infty\left[\rightarrow \mathbb{R}_{+}\right.\right.\right.\right.$is given by

$$
\Lambda(s, t)=\frac{\Gamma(s, t)}{\int_{t_{0}}^{t} \Gamma(u, t) d u},
$$

where the quantity $\Gamma(s, t)$ is defined by (2). We then obtain the following consequence of Proposition 4.1.

Corollary 4.2. Let $S$ be a nonempty subset of $\mathcal{H}$, and let $x:\left[t_{0},+\infty[\rightarrow \mathcal{H}\right.$ be a continuous map, supposed to be bounded on $\left[t_{0},+\infty\left[\right.\right.$. Given a continuous function $\gamma:\left[t_{0},+\infty\left[\rightarrow \mathbb{R}_{+}\right.\right.$satisfying $\left(H_{0}\right)$, let $\Gamma(s)$ and $\Gamma(s, t)$ be the quantities defined respectively by (1) and (2). Suppose that $\int_{t_{0}}^{+\infty} \Gamma(s) d s=+\infty$. Let $\widehat{x}:\left[t_{0},+\infty[\rightarrow \mathcal{H}\right.$ be the averaged map defined by

$$
\widehat{x}(t)=\frac{1}{\int_{t_{0}}^{t} \Gamma(s, t) d s} \int_{t_{0}}^{t} \Gamma(s, t) x(s) d s .
$$

Assume that

(i) for every $z \in S, \lim _{t \rightarrow+\infty}\|x(t)-z\|$ exists;

(ii) every weak sequential limit point of $\widehat{x}(t)$, as $t \rightarrow+\infty$, belongs to $S$.

Then $\widehat{x}(t)$ converges weakly as $t \rightarrow+\infty$ to a point in $S$. 
Proof. Just check that conditions (19) and (20) of Proposition 4.1 are satisfied for the function $\Lambda$ : $\left[t_{0},+\infty\left[\times\left[t_{0},+\infty\left[\rightarrow \mathbb{R}_{+}\right.\right.\right.\right.$given by (22). Property (19) clearly holds true. Observe that for every $T \geq t_{0}$,

$$
\int_{t_{0}}^{T} \Lambda(s, t) d s=\frac{\int_{t_{0}}^{T} \Gamma(s, t) d s}{\int_{t_{0}}^{t} \Gamma(u, t) d u} \leq \frac{\int_{t_{0}}^{T} \Gamma(s) d s}{\int_{t_{0}}^{t} \Gamma(u, t) d u} .
$$

The quantity $\int_{t_{0}}^{T} \Gamma(s) d s$ is finite and independent of $t$. On the other hand, from the assumption $\int_{t_{0}}^{+\infty} \Gamma(s) d s=+\infty$ we deduce that $\lim _{t \rightarrow+\infty} \int_{t_{0}}^{t} \Gamma(u, t) d u=+\infty$, see (3). We deduce from the above inequality that $\lim _{t \rightarrow+\infty} \int_{t_{0}}^{T} \Lambda(s, t) d s=0$, hence property (20) is satisfied. It ensues that Proposition 4.1 can be applied, which ends the proof.

4.2. Ergodic convergence of the trajectories. To each solution $x($.$) of (RIMS) { }_{\gamma, \lambda}$, we associate the averaged trajectory $\widehat{x}($.$) defined by$

$$
\widehat{x}(t)=\frac{1}{\int_{t_{0}}^{t} \Gamma(s, t) d s} \int_{t_{0}}^{t} \Gamma(s, t) x(s) d s .
$$

We show that under suitable conditions, every averaged trajectory $\widehat{x}($.$) converges weakly as t \rightarrow+\infty$ toward some zero of the operator $A$.

Theorem 4.3. Let $A: \mathcal{H} \rightarrow 2^{\mathcal{H}}$ be a maximally monotone operator such that $\operatorname{zer} A \neq \emptyset$ and let $\lambda:\left[t_{0},+\infty\left[\rightarrow \mathbb{R}_{+}^{*}\right.\right.$ be a differentiable function. Suppose that the differentiable function $\gamma:\left[t_{0},+\infty\left[\rightarrow \mathbb{R}_{+}\right.\right.$ satisfies $\left(H_{0}\right)$. For $s, t \geq t_{0}$, let $\Gamma(s)$ and $\Gamma(s, t)$ be the quantities respectively defined by (1) and (2). Assume that conditions $\left(H_{1}\right)-\left(H_{2}\right)-\left(H_{3}\right)$ hold, together with $\int_{t_{0}}^{+\infty} \Gamma(s) d s=+\infty$. Then for any global solution $x($.$) of (\mathrm{RIMS})_{\gamma, \lambda}$, there exists $x_{\infty} \in \operatorname{zer} A$ such that

$$
\widehat{x}(t)=\frac{1}{\int_{t_{0}}^{t} \Gamma(s, t) d s} \int_{t_{0}}^{t} \Gamma(s, t) x(s) d s \rightarrow x_{\infty} \quad \text { weakly in } \mathcal{H} \text { as } t \rightarrow+\infty .
$$

Proof. We apply Corollary 4.2 with $S=\operatorname{zer} A$. Condition $(i)$ of Corollary 4.2 is realized in view of Theorem 2.3 (iii). Let us now assume that there exist $x_{\infty} \in \mathcal{H}$ and a sequence $\left(t_{n}\right)$ such that $t_{n} \rightarrow+\infty$ and $\widehat{x}\left(t_{n}\right) \rightarrow x_{\infty}$ weakly in $\mathcal{H}$ as $n \rightarrow+\infty$. Let us fix $(z, q) \in \operatorname{gph} A$ and define the function $h:\left[t_{0},+\infty\left[\rightarrow \mathbb{R}_{+}\right.\right.$ by $h(t)=\frac{1}{2}\|x(t)-z\|^{2}$. Since $q \in A(z)$ and $A_{\lambda(t)}(x(t)) \in A\left(x(t)-\lambda(t) A_{\lambda(t)}(x(t))\right)$, the monotonicity of $A$ implies that

$$
\left\langle x(t)-\lambda(t) A_{\lambda(t)}(x(t))-z, A_{\lambda(t)}(x(t))-q\right\rangle \geq 0,
$$

hence

$$
\begin{aligned}
\left\langle x(t)-z, A_{\lambda(t)}(x(t))\right\rangle & \geq \lambda(t)\left\|A_{\lambda(t)}(x(t))\right\|^{2}+\left\langle x(t)-\lambda(t) A_{\lambda(t)}(x(t))-z, q\right\rangle \\
& \geq\left\langle x(t)-\lambda(t) A_{\lambda(t)}(x(t))-z, q\right\rangle .
\end{aligned}
$$

Recalling equality (4), we obtain for every $t \geq t_{0}$,

$$
\ddot{h}(t)+\gamma(t) \dot{h}(t) \leq\|\dot{x}(t)\|^{2}-\left\langle x(t)-\lambda(t) A_{\lambda(t)}(x(t))-z, q\right\rangle .
$$

Using Lemma B.1 $(i)$ with $g(t)=\|\dot{x}(t)\|^{2}-\left\langle x(t)-\lambda(t) A_{\lambda(t)}(x(t))-z, q\right\rangle$, we obtain for every $t \geq t_{0}$,

$$
h(t) \leq h\left(t_{0}\right)+\dot{h}\left(t_{0}\right)\left(\int_{t_{0}}^{t} \frac{d u}{p(u)}\right)+\int_{t_{0}}^{t} \Gamma(s, t)\left[\|\dot{x}(s)\|^{2}-\left\langle x(s)-\lambda(s) A_{\lambda(s)}(x(s))-z, q\right\rangle\right] d s .
$$

Since $h(t) \geq 0$ and $\Gamma(s, t) \leq \Gamma(s)$, we deduce that

$$
\int_{t_{0}}^{t} \Gamma(s, t)\left\langle x(s)-\lambda(s) A_{\lambda(s)}(x(s))-z, q\right\rangle d s \leq h\left(t_{0}\right)+\dot{h}\left(t_{0}\right)\left(\int_{t_{0}}^{t} \frac{d u}{p(u)}\right)+\int_{t_{0}}^{t} \Gamma(s)\|\dot{x}(s)\|^{2} d s .
$$


Recalling the assumption $\int_{t_{0}}^{+\infty} \frac{d u}{p(u)}<+\infty$ and the estimate $\int_{t_{0}}^{+\infty} \Gamma(s)\|\dot{x}(s)\|^{2} d s<+\infty$ (see Theorem $2.3(i)$ ), we infer that for every $t \geq t_{0}$,

$$
\int_{t_{0}}^{t} \Gamma(s, t)\left\langle x(s)-\lambda(s) A_{\lambda(s)}(x(s))-z, q\right\rangle d s \leq C,
$$

where we have set

$$
C:=h\left(t_{0}\right)+\left|\dot{h}\left(t_{0}\right)\right|\left(\int_{t_{0}}^{+\infty} \frac{d u}{p(u)}\right)+\int_{t_{0}}^{+\infty} \Gamma(s)\|\dot{x}(s)\|^{2} d s
$$

It ensues that

$$
\begin{aligned}
\int_{t_{0}}^{t} \Gamma(s, t)\langle x(s)-z, q\rangle d s & \leq C+\int_{t_{0}}^{t} \Gamma(s, t)\left\langle\lambda(s) A_{\lambda(s)}(x(s)), q\right\rangle d s \\
& \leq C+\|q\| \int_{t_{0}}^{t} \Gamma(s, t) \lambda(s)\left\|A_{\lambda(s)}(x(s))\right\| d s .
\end{aligned}
$$

This can be rewritten as

$$
\left\langle\int_{t_{0}}^{t} \Gamma(s, t)(x(s)-z) d s, q\right\rangle \leq C+\|q\| \int_{t_{0}}^{t} \Gamma(s, t) \lambda(s)\left\|A_{\lambda(s)}(x(s))\right\| d s .
$$

Dividing by $\int_{t_{0}}^{t} \Gamma(s, t) d s$, we find

$$
\langle\widehat{x}(t)-z, q\rangle \leq \frac{C}{\int_{t_{0}}^{t} \Gamma(s, t) d s}+\frac{\|q\|}{\int_{t_{0}}^{t} \Gamma(s, t) d s} \int_{t_{0}}^{t} \Gamma(s, t) \lambda(s)\left\|A_{\lambda(s)}(x(s))\right\| d s .
$$

The assumption $\int_{t_{0}}^{+\infty} \Gamma(s) d s=+\infty$ implies that $\lim _{t \rightarrow+\infty} \int_{t_{0}}^{t} \Gamma(s, t) d s=+\infty$, see (3). On the other hand, we have $\lim _{t \rightarrow+\infty} \lambda(t) A_{\lambda(t)}(x(t))=0$ by Theorem $2.3(v)$. From the Cesaro property, we infer that

$$
\frac{1}{\int_{t_{0}}^{t} \Gamma(s, t) d s} \int_{t_{0}}^{t} \Gamma(s, t) \lambda(s)\left\|A_{\lambda(s)}(x(s))\right\| d s \rightarrow 0 \quad \text { as } t \longrightarrow+\infty,
$$

see Lemma B.4 (ii). Taking the limit as $t \rightarrow+\infty$ in inequality (24), we then obtain

$$
\limsup _{t \rightarrow+\infty}\langle\widehat{x}(t)-z, q\rangle \leq 0 .
$$

Recall that the sequence $\left(t_{n}\right)$ is such that $\widehat{x}\left(t_{n}\right) \rightarrow x_{\infty}$ weakly in $\mathcal{H}$ as $n \rightarrow+\infty$, hence $\left\langle\widehat{x}\left(t_{n}\right)-z, q\right\rangle \rightarrow$ $\left\langle x_{\infty}-z, q\right\rangle$ as $n \rightarrow+\infty$. From what precedes, we deduce that $\left\langle x_{\infty}-z, q\right\rangle \leq 0$ for every $(z, q) \in \operatorname{gph} A$. Since the operator $A$ is maximally monotone, we infer that $0 \in A\left(x_{\infty}\right)$. We have proved that $x_{\infty} \in \operatorname{zer} A$, which shows that condition (ii) of Corollary 4.2 is satisfied.

Let us now consider the alternate averaged trajectory $\bar{x}$ defined by

$$
\bar{x}(t)=\frac{1}{\int_{t_{0}}^{t} \Gamma(s) d s} \int_{t_{0}}^{t} \Gamma(s) x(s) d s,
$$

for every $t \geq t_{0}$. The next result gives sufficient conditions that ensure the weak convergence of $\bar{x}(t)$ as $t \rightarrow+\infty$ toward a zero of $A$.

Theorem 4.4. Under the hypotheses of Theorem 4.3, assume moreover that

$$
\left(\int_{t}^{+\infty} \frac{d s}{p(s)}\right)\left(\int_{t_{0}}^{t} p(s) d s\right)=o\left(\int_{t_{0}}^{t} \Gamma(s) d s\right) \quad \text { as } t \rightarrow+\infty .
$$


Then for any global solution $x($.$) of (RIMS) { }_{\gamma, \lambda}$, there exists $x_{\infty} \in \operatorname{zer} A$ such that

$$
\bar{x}(t)=\frac{1}{\int_{t_{0}}^{t} \Gamma(s) d s} \int_{t_{0}}^{t} \Gamma(s) x(s) d s \rightarrow x_{\infty} \quad \text { weakly in } \mathcal{H} \text { as } t \rightarrow+\infty .
$$

The latter result still holds true if the function $\Gamma$ in the above quotient is replaced with a function $\widetilde{\Gamma}$ : $\left[t_{0},+\infty\left[\rightarrow \mathbb{R}_{+}\right.\right.$such that $\widetilde{\Gamma}(s) \sim \Gamma(s)$ as $s \rightarrow+\infty$.

Proof. We are going to show that $\lim _{t \rightarrow+\infty}\|\widehat{x}(t)-\bar{x}(t)\|=0$, where $\widehat{x}$ is the averaged trajectory of Theorem 4.3. For that purpose, we use Lemma B.5 with the functions $\Lambda_{1}, \Lambda_{2}:\left[t_{0},+\infty\left[\times\left[t_{0},+\infty\left[\rightarrow \mathbb{R}_{+}\right.\right.\right.\right.$ respectively defined by

$$
\Lambda_{1}(s, t)=\frac{\Gamma(s, t)}{\int_{t_{0}}^{t} \Gamma(u, t) d u}, \quad \Lambda_{2}(s, t)=\frac{\Gamma(s)}{\int_{t_{0}}^{t} \Gamma(u) d u}, \quad \text { if } s \leq t,
$$

and $\Lambda_{1}(s, t)=\Lambda_{2}(s, t)=0$ if $s>t$. The functions $\Lambda_{1}$ and $\Lambda_{2}$ clearly satisfy property (19). Let us now check that

$$
\lim _{t \rightarrow+\infty} \int_{t_{0}}^{+\infty}\left|\Lambda_{1}(s, t)-\Lambda_{2}(s, t)\right| d s=0
$$

For $s \leq t$, we have

$$
\Lambda_{1}(s, t)-\Lambda_{2}(s, t)=\frac{\Gamma(s, t)}{\int_{t_{0}}^{t} \Gamma(u, t) d u} \frac{\int_{t_{0}}^{t}(\Gamma(u)-\Gamma(u, t)) d u}{\int_{t_{0}}^{t} \Gamma(u) d u}+\frac{\Gamma(s, t)-\Gamma(s)}{\int_{t_{0}}^{t} \Gamma(u) d u}
$$

and hence

$$
\left|\Lambda_{1}(s, t)-\Lambda_{2}(s, t)\right| \leq \frac{\Gamma(s, t)}{\int_{t_{0}}^{t} \Gamma(u, t) d u} \frac{\int_{t_{0}}^{t}|\Gamma(u)-\Gamma(u, t)| d u}{\int_{t_{0}}^{t} \Gamma(u) d u}+\frac{|\Gamma(s, t)-\Gamma(s)|}{\int_{t_{0}}^{t} \Gamma(u) d u} .
$$

By integrating on $\left[t_{0}, t\right]$, we find

$$
\int_{t_{0}}^{t}\left|\Lambda_{1}(s, t)-\Lambda_{2}(s, t)\right| d s \leq 2 \frac{\int_{t_{0}}^{t}|\Gamma(s, t)-\Gamma(s)| d s}{\int_{t_{0}}^{t} \Gamma(s) d s} .
$$

Recalling that $\Lambda_{1}(s, t)=\Lambda_{2}(s, t)=0$ for $s>t$, this implies that

$$
\int_{t_{0}}^{+\infty}\left|\Lambda_{1}(s, t)-\Lambda_{2}(s, t)\right| d s \leq 2 \frac{\int_{t_{0}}^{t}|\Gamma(s, t)-\Gamma(s)| d s}{\int_{t_{0}}^{t} \Gamma(s) d s} .
$$

From the expression of $\Gamma(s)$ and $\Gamma(s, t)$, see (1) and (2), we immediately deduce that

$$
\int_{t_{0}}^{+\infty}\left|\Lambda_{1}(s, t)-\Lambda_{2}(s, t)\right| d s \leq 2 \frac{\left(\int_{t}^{+\infty} \frac{d s}{p(s)}\right)\left(\int_{t_{0}}^{t} p(s) d s\right)}{\int_{t_{0}}^{t} \Gamma(s) d s} .
$$

In view of assumption (25), we then obtain (26). By applying Lemma B.5, we infer that $\lim _{t \rightarrow+\infty} \| \widehat{x}(t)-$ $\bar{x}(t) \|=0$. On the other hand, Theorem 4.3 shows that there exists $x_{\infty} \in \operatorname{zer} A$ such that $\widehat{x}(t) \rightarrow x_{\infty}$ weakly in $\mathcal{H}$ as $t \rightarrow+\infty$. We then conclude that $\bar{x}(t) \rightarrow x_{\infty}$ weakly in $\mathcal{H}$ as $t \rightarrow+\infty$.

Now assume that the function $\widetilde{\Gamma}:\left[t_{0},+\infty\left[\rightarrow \mathbb{R}_{+}\right.\right.$is such that $\widetilde{\Gamma}(s) \sim \Gamma(s)$ as $s \rightarrow+\infty$. Let us denote by $\widetilde{\Lambda}_{2}$ the function defined by

$$
\widetilde{\Lambda}_{2}(s, t)=\frac{\widetilde{\Gamma}(s)}{\int_{t_{0}}^{t} \widetilde{\Gamma}(u) d u} \quad \text { if } s \leq t
$$


and $\widetilde{\Lambda}_{2}(s, t)=0$ if $s>t$. The corresponding averaged trajectory is denoted by $\widetilde{x}$. By arguing as above, we obtain that

$$
\int_{t_{0}}^{+\infty}\left|\widetilde{\Lambda}_{2}(s, t)-\Lambda_{2}(s, t)\right| d s \leq 2 \frac{\int_{t_{0}}^{t}|\widetilde{\Gamma}(s)-\Gamma(s)| d s}{\int_{t_{0}}^{t} \Gamma(s) d s} .
$$

Then, using the estimate

$$
\int_{t_{0}}^{t}|\widetilde{\Gamma}(s)-\Gamma(s)| d s=o\left(\int_{t_{0}}^{t} \Gamma(s) d s\right) \quad \text { as } t \rightarrow+\infty,
$$

we deduce that

$$
\int_{t_{0}}^{+\infty}\left|\widetilde{\Lambda}_{2}(s, t)-\Lambda_{2}(s, t)\right| d s \longrightarrow 0 \quad \text { as } t \rightarrow+\infty .
$$

In view of Lemma B.5, this implies that $\lim _{t \rightarrow+\infty}\|\widetilde{x}(t)-\bar{x}(t)\|=0$, which ends the proof.

Let us now apply Theorem 4.4 to the class of differentiable functions $\gamma, \lambda:\left[t_{0},+\infty\left[\rightarrow \mathbb{R}_{+}^{*}\right.\right.$ satisfying

$$
\lim _{t \rightarrow+\infty} \frac{\dot{\gamma}(t)}{\gamma(t)^{2}}=0 \quad \text { and } \quad \lim _{t \rightarrow+\infty} \frac{\frac{d}{d t}(\lambda(t) \gamma(t))}{\lambda(t) \gamma(t)^{2}}=0
$$

Corollary 4.5. Let $A: \mathcal{H} \rightarrow 2^{\mathcal{H}}$ be a maximally monotone operator such that zer $A \neq \emptyset$. Let $\gamma$, $\lambda:\left[t_{0},+\infty\left[\rightarrow \mathbb{R}_{+}^{*}\right.\right.$ be two differentiable functions satisfying conditions (27). Assume that

(a) $\liminf _{t \rightarrow+\infty} \lambda(t) \gamma(t)^{2}>1$;

(b) $|\dot{\lambda}(t)|=\mathcal{O}(1 / \gamma(t))$ as $t \rightarrow+\infty$;

(c) $\int_{t_{0}}^{+\infty} \frac{d s}{\lambda(s) \gamma(s)}=+\infty$

(d) $\int_{t_{0}}^{+\infty} \frac{d s}{\gamma(s)}=+\infty$.

Then for any global solution $x($.$) of (RIMS) { }_{\gamma, \lambda}$, there exists $x_{\infty} \in \operatorname{zer} A$ such that

$$
\frac{1}{\int_{t_{0}}^{t} \frac{d s}{\gamma(s)}} \int_{t_{0}}^{t} \frac{x(s)}{\gamma(s)} d s \rightarrow x_{\infty} \quad \text { weakly in } \mathcal{H} \text { as } t \rightarrow+\infty .
$$

Proof. Let us check that the assumptions of Theorem 4.4 are satisfied. Assumption $\left(H_{0}\right)$ is verified in view of Proposition $3.1(i)$ applied with $c=0$. Since $\liminf _{t \rightarrow+\infty} \lambda(t) \gamma(t)^{2}>1$, condition $\left(H_{1}\right)$ holds true by Proposition 3.2 used with $c=c^{\prime}=0$. On the other hand, Proposition 3.1 shows that

$$
\int_{t}^{+\infty} \frac{d s}{p(s)} \sim \frac{1}{p(t) \gamma(t)} \quad \text { and } \quad \int_{t_{0}}^{t} \frac{p(s)}{\lambda(s)} d s \sim \frac{p(t)}{\lambda(t) \gamma(t)} \quad \text { as } t \rightarrow+\infty,
$$

thus implying that $\Gamma(t) \sim \frac{1}{\gamma(t)}$ as $t \rightarrow+\infty$. It ensues that the first condition of $\left(H_{2}\right)$ is automatically satisfied, while the second one is given by $(b)$. Condition $\left(H_{3}\right)$ is implied by the assumption $(c)$. In the same way, condition $\int_{t_{0}}^{+\infty} \Gamma(s) d s=+\infty$ is guaranteed by the assumption $(d)$. It remains to establish condition (25) of Theorem 4.4. By applying Proposition $3.1(i i)$ with $\lambda(t) \equiv 1$ and $c^{\prime}=0$, we obtain

$$
\int_{t_{0}}^{t} p(s) d s \sim \frac{p(t)}{\gamma(t)} \quad \text { as } t \rightarrow+\infty
$$

In view of the first equivalence of $(28)$, we infer that

$$
\left(\int_{t}^{+\infty} \frac{d s}{p(s)}\right)\left(\int_{t_{0}}^{t} p(s) d s\right) \sim \frac{1}{\gamma(t)^{2}} \quad \text { as } t \rightarrow+\infty .
$$


It ensues that condition (25) amounts to $\frac{1}{\gamma(t)^{2}}=o\left(\int_{t_{0}}^{t} \Gamma(s) d s\right)$ as $t \rightarrow+\infty$, which is in turn equivalent to

$$
\frac{1}{\gamma(t)^{2}}=o\left(\int_{t_{0}}^{t} \frac{d s}{\gamma(s)}\right) \quad \text { as } t \rightarrow+\infty \text {. }
$$

Since $\lim _{t \rightarrow+\infty} \dot{\gamma}(t) / \gamma(t)^{2}=0$, we have $-\dot{\gamma}(t) / \gamma(t)^{3}=o(1 / \gamma(t))$ as $t \rightarrow+\infty$. By integrating on $\left[t_{0}, t\right]$, we obtain

$$
\left[\frac{1}{2 \gamma(t)^{2}}\right]_{t_{0}}^{t}=\int_{t_{0}}^{t}-\frac{\dot{\gamma}(s)}{\gamma(s)^{3}} d s=o\left(\int_{t_{0}}^{t} \frac{d s}{\gamma(s)}\right) \quad \text { as } t \rightarrow+\infty
$$

because $\int_{t_{0}}^{+\infty} \frac{d s}{\gamma(s)}=+\infty$ by assumption. It ensues that condition (29) is fulfilled, hence all the hypotheses of Theorem 4.4 are satisfied. We deduce that there exists $x_{\infty} \in \operatorname{zer} A$ such that

$$
\frac{1}{\int_{t_{0}}^{t} \Gamma(s) d s} \int_{t_{0}}^{t} \Gamma(s) x(s) d s \rightarrow x_{\infty} \quad \text { weakly in } \mathcal{H} \text { as } t \rightarrow+\infty .
$$

Since $\Gamma(t) \sim 1 / \gamma(t)$ as $t \rightarrow+\infty$, the above result holds true with the function $1 / \gamma$ in place of $\Gamma$, see the last assertion of Theorem 4.4.

Let us now particularize to the case $\gamma(t)=\alpha t^{q}$ and $\lambda(t)=\beta t^{r}$, for some $\left.\left.\alpha, \beta>0, q \in\right]-1,1\right]$ and $r \in \mathbb{R}$.

Corollary 4.6. Let $A: \mathcal{H} \rightarrow 2^{\mathcal{H}}$ be a maximally monotone operator such that zer $A \neq \emptyset$. Assume that $\gamma(t)=\alpha t^{q}$ and $\lambda(t)=\beta t^{r}$ for every $t \geq t_{0}>0$. Let $\left.\left.(q, r) \in\right]-1,1\right] \times \mathbb{R}$ be such that $q+r \leq 1$ and $2 q+r \geq 0$, and let $(\alpha, \beta) \in \mathbb{R}_{+}^{*} \times \mathbb{R}_{+}^{*}$ be such that $\alpha^{2} \beta>1$ if $2 q+r=0$ (no condition if $2 q+r>0$ ).

Then for any global solution $x($.$) of (RIMS )_{\gamma, \lambda}$, there exists $x_{\infty} \in$ zer $A$ such that

$$
\frac{1}{\int_{t_{0}}^{t} \frac{d s}{s^{q}}} \int_{t_{0}}^{t} \frac{x(s)}{s^{q}} d s \rightarrow x_{\infty} \quad \text { weakly in } \mathcal{H} \text { as } t \rightarrow+\infty .
$$

Proof. The conditions of (27) are guaranteed by $q>-1$. On the other hand, we have $\lambda(t) \gamma(t)^{2}=$ $\alpha^{2} \beta t^{2 q+r}$, hence

$$
\lim _{t \rightarrow+\infty} \lambda(t) \gamma(t)^{2}=\left\{\begin{array}{lll}
+\infty & \text { if } & 2 q+r>0 \\
\alpha^{2} \beta & \text { if } & 2 q+r=0
\end{array}\right.
$$

It follows that the condition $\liminf _{t \rightarrow+\infty} \lambda(t) \gamma(t)^{2}>1$ is ensured by the hypotheses of Corollary 4.6. Conditions $|\dot{\lambda}(t)|=\mathcal{O}(1 / \gamma(t))$ as $t \rightarrow+\infty$, and $\int_{t_{0}}^{+\infty} \frac{d s}{\lambda(s) \gamma(s)}=+\infty$ amount respectively to $q+r \leq 1$, which holds true by assumption. The condition $\int_{t_{0}}^{+\infty} \frac{d s}{\gamma(s)}=+\infty$ is implied by $q \leq 1$. Then just apply Corollary 4.5 .

\section{PART C: THE SUBDIFFERENTIAL CASE}

Let us particularize our study to the case $A=\partial \Phi$, where $\Phi: \mathcal{H} \rightarrow \mathbb{R} \cup\{+\infty\}$ is a convex lower semicontinuous proper function. Then $A_{\lambda}=\nabla \Phi_{\lambda}$ is equal to the gradient of $\Phi_{\lambda}: \mathcal{H} \rightarrow \mathbb{R}$, which is the Moreau envelope of $\Phi$ of index $\lambda>0$. Let us recall that, for all $x \in \mathcal{H}$

$$
\Phi_{\lambda}(x)=\inf _{\xi \in \mathcal{H}}\left\{\Phi(\xi)+\frac{1}{2 \lambda}\|x-\xi\|^{2}\right\} .
$$


In this case, we will study the rate of convergence of the values, when the time $t$ goes to $+\infty$, of the trajectories of the second-order differential equation

$$
(\mathrm{RIGS})_{\gamma, \lambda} \quad \ddot{x}(t)+\gamma(t) \dot{x}(t)+\nabla \Phi_{\lambda(t)}(x(t))=0,
$$

called the Regularized Inertial Gradient System with parameters $\gamma, \lambda$. As a main feature, the above system involves two time-dependent positive parameters: the Moreau regularization parameter $\lambda(t)$, and the damping parameter $\gamma(t)$. System (RIGS) ${ }_{\gamma, \lambda}$ comes as a natural development of several recent studies concerning fast inertial dynamics and algorithms for convex optimization. Indeed, when $\Phi$ is a smooth convex function, it was highlighted that the fact of taking a vanishing damping coefficient $\gamma(t)$ in system

$$
(\mathrm{IGS})_{\gamma} \quad \ddot{x}(t)+\gamma(t) \dot{x}(t)+\nabla \Phi(x(t))=0,
$$

is a key property for obtaining fast optimization methods. Precisely Su, Boyd and Candès [28] showed that, in the particular case $\gamma(t)=\frac{3}{t}$, (IGS) $\gamma$ is a continuous version of the fast gradient method initiated by Nesterov [23], with $\Phi(x(t))-\min _{\mathcal{H}} \Phi=\mathcal{O}\left(\frac{1}{t^{2}}\right)$ in the worst case. Attouch and Peypouquet [7] and May [22] have improved this result by showing that $\Phi(x(t))-\min _{\mathcal{H}} \Phi=o\left(\frac{1}{t^{2}}\right)$ for $\gamma(t)=\frac{\alpha}{t}$ with $\alpha>3$. Recently, in the case of a general damping function $\gamma(\cdot)$, the study of the speed of convergence of trajectories of (IGS) $)_{\gamma}$ was developed by Attouch-Cabot in [3]. Note that a main advantage of (RIGS) ${ }_{\gamma, \lambda}$ over (IGS) ${ }_{\gamma}$ is that $\Phi$ is just assumed to be lower semicontinuous (not necessarily smooth). In line with these results, by jointly adjusting the tuning of the two parameters in (RIGS) ${ }_{\gamma, \lambda}$, we will obtain fast convergence results for the values.

\section{Convergence RAtes AND WEAK CONVERGEnCE OF THE TRAJECTORIES}

The following assumptions and notations will be needed throughout this section:



5.1. Preliminaries on Moreau envelopes. For classical facts about the Moreau envelopes we refer the reader to $[11,15,25,27]$. We point out the following properties that will be useful in the sequel:

(i) $\lambda \in] 0,+\infty\left[\mapsto \Phi_{\lambda}(x)\right.$ is nonincreasing for all $x \in \mathcal{H}$;

(ii) $\inf _{\mathcal{H}} \Phi=\inf _{\mathcal{H}} \Phi_{\lambda}$ for all $\lambda>0$;

(iii) $\operatorname{argmin} \Phi=\operatorname{argmin} \Phi_{\lambda}$ for all $\lambda>0$.

It turns out that it is convenient to consider the Moreau envelope as a function of the two variables $x \in \mathcal{H}$ and $\lambda \in] 0,+\infty$ [. Its differentiability properties with respect to $(x, \lambda)$ play a crucial role in our analysis.

a. Let us first recall some classical facts concerning the differentiability properties with respect to $x$ of the Moreau envelope $x \mapsto \Phi_{\lambda}(x)$. The infimum in (30) is achieved at a unique point

$$
\operatorname{prox}_{\lambda \Phi}(x)=\operatorname{argmin}_{\xi \in \mathcal{H}}\left\{\Phi(\xi)+\frac{1}{2 \lambda}\|x-\xi\|^{2}\right\},
$$

which gives

$$
\Phi_{\lambda}(x)=\Phi\left(\operatorname{prox}_{\lambda \Phi}(x)\right)+\frac{1}{2 \lambda}\left\|x-\operatorname{prox}_{\lambda \Phi}(x)\right\|^{2} .
$$

Writing the optimality condition for (31), we get

$$
\operatorname{prox}_{\lambda \Phi}(x)+\lambda \partial \Phi\left(\operatorname{prox}_{\lambda \Phi}(x)\right) \ni x,
$$


that is

$$
\operatorname{prox}_{\lambda \Phi}(x)=(I+\lambda \partial \Phi)^{-1}(x) .
$$

Thus, $\operatorname{prox}_{\lambda \Phi}$ is the resolvent of index $\lambda>0$ of the maximally monotone operator $\partial \Phi$. As a consequence, the mapping $\operatorname{prox}_{\lambda \Psi}: \mathcal{H} \rightarrow \mathcal{H}$ is firmly expansive. For any $\lambda>0$, the function $x \mapsto \Phi_{\lambda}(x)$ is continuously differentiable, with

Equivalently

$$
\nabla \Phi_{\lambda}(x)=\frac{1}{\lambda}\left(x-\operatorname{prox}_{\lambda \Phi}(x)\right)
$$

$$
\nabla \Phi_{\lambda}=\frac{1}{\lambda}\left(I-(I+\lambda \partial \Phi)^{-1}\right)=(\partial \Phi)_{\lambda}
$$

which is the Yosida approximation of the maximally monotone operator $\partial \Phi$. As such, $\nabla \Phi_{\lambda}$ is Lipschitz continuous, with Lipschitz constant $\frac{1}{\lambda}$, and $\Phi_{\lambda} \in \mathcal{C}^{1,1}(\mathcal{H})$.

b. A less known result is the $\mathcal{C}^{1}$-regularity of the function $\lambda \mapsto \Phi_{\lambda}(x)$, for any $x \in \mathcal{H}$. Its derivative is given by

$$
\frac{d}{d \lambda} \Phi_{\lambda}(x)=-\frac{1}{2}\left\|\nabla \Phi_{\lambda}(x)\right\|^{2}
$$

This result is known as the Lax-Hopf formula for the above first-order Hamilton-Jacobi equation, see [2, Remark 3.32; Lemma 3.27], and [21]. A proof is given in Lemma A.1 for the convenience of the reader. As a consequence of the semi-group property satisfied by the orbits of the autonomous evolution equation (32), for any $x \in \mathcal{H}, \lambda>0$ and $\mu>0$,

$$
\left(\Phi_{\lambda}\right)_{\mu}(x)=\Phi_{(\lambda+\mu)}(x)
$$

5.2. Preliminary estimates. Let us introduce functions $W, h_{z}$, of constant use in this section.

5.2.1. Global energy. The global energy of the system $W:\left[t_{0},+\infty\left[\rightarrow \mathbb{R}_{+}\right.\right.$is given by

$$
W(t)=\frac{1}{2}\|\dot{x}(t)\|^{2}+\Phi_{\lambda(t)}(x(t))-\min _{\mathcal{H}} \Phi .
$$

Since $\inf _{\mathcal{H}} \Phi=\inf _{\mathcal{H}} \Phi_{\lambda}$, we have $W \geq 0$. From (RIGS) ${ }_{\gamma, \lambda}$ and property (32), we immediately obtain the following equality

$$
\dot{W}(t)=-\gamma(t)\|\dot{x}(t)\|^{2}-\frac{\dot{\lambda}(t)}{2}\left\|\nabla \Phi_{\lambda(t)}(x(t))\right\|^{2} .
$$

As a direct consequence of (34), we obtain the following results.

Proposition 5.1. The function $W$ is nonincreasing, and hence $W_{\infty}:=\lim _{t \rightarrow+\infty} W(t)$ exists. In addition,

$$
\sup _{t \geq t_{0}}\|\dot{x}(t)\|<+\infty, \quad \int_{t_{0}}^{\infty} \gamma(t)\|\dot{x}(t)\|^{2} d t<+\infty \quad \text { and } \quad \int_{t_{0}}^{\infty} \dot{\lambda}(t)\left\|\nabla \Phi_{\lambda(t)}(x(t))\right\|^{2} d t<+\infty .
$$

Proof. From (34), and $\lambda$ nondecreasing, we deduce that $\dot{W}(t) \leq 0$. Hence, $W$ is nonincreasing. Since $W$ is nonnegative, $W_{\infty}:=\lim _{t \rightarrow+\infty} W(t)$ exists. After integrating $(34)$ from $t_{0}$ to $t$, we get

$$
W(t)-W\left(t_{0}\right)+\int_{t_{0}}^{t} \gamma(s)\|\dot{x}(s)\|^{2} d s+\frac{1}{2} \int_{t_{0}}^{t} \dot{\lambda}(s)\left\|\nabla \Phi_{\lambda(s)}(x(s))\right\|^{2} d s \leq 0 .
$$

By definition of $W$, and using again that $\inf _{\mathcal{H}} \Phi=\inf _{\mathcal{H}} \Phi_{\lambda}$, it follows that

$$
\frac{1}{2}\|\dot{x}(t)\|^{2}+\int_{t_{0}}^{t} \gamma(s)\|\dot{x}(s)\|^{2} d s+\frac{1}{2} \int_{t_{0}}^{t} \dot{\lambda}(s)\left\|\nabla \Phi_{\lambda(s)}(x(s))\right\|^{2} d s \leq W\left(t_{0}\right) .
$$

This being true for any $t \geq t_{0}$, we get the conclusion. 
5.2.2. Anchor. Given $z \in \mathcal{H}$, we define $h_{z}:\left[t_{0},+\infty[\rightarrow \mathbb{R}\right.$ by

$$
h_{z}(t)=\frac{1}{2}\|x(t)-z\|^{2} \text {. }
$$

We have the following:

Lemma 5.2. For each $z \in \mathcal{H}$ and all $t \geq t_{0}$, we have

$$
\begin{aligned}
& \ddot{h}_{z}(t)+\gamma(t) \dot{h}_{z}(t)+\left\langle x(t)-z, \nabla \Phi_{\lambda(t)}(x(t))\right\rangle=\|\dot{x}(t)\|^{2} \\
& \ddot{h}_{z}(t)+\gamma(t) \dot{h}_{z}(t)+\Phi_{\lambda(t)}(x(t))-\Phi_{\lambda(t)}(z) \leq\|\dot{x}(t)\|^{2} .
\end{aligned}
$$

In particular, if $z \in \operatorname{argmin} \Phi$, then

$$
\ddot{h}_{z}(t)+\gamma(t) \dot{h}_{z}(t) \leq\|\dot{x}(t)\|^{2} .
$$

Proof. First observe that

$$
\dot{h}_{z}(t)=\langle x(t)-z, \dot{x}(t)\rangle \quad \text { and } \quad \ddot{h}_{z}(t)=\langle x(t)-z, \ddot{x}(t)\rangle+\|\dot{x}(t)\|^{2} .
$$

By $(\text { RIGS })_{\gamma, \lambda}$ and the convexity of $\Phi_{\lambda(t)}$, it ensues that

$$
\ddot{h}_{z}(t)+\gamma(t) \dot{h}_{z}(t)=\|\dot{x}(t)\|^{2}+\left\langle x(t)-z,-\nabla \Phi_{\lambda(t)}(x(t))\right\rangle \leq\|\dot{x}(t)\|^{2}+\Phi_{\lambda(t)}(z)-\Phi_{\lambda(t)}(x(t)),
$$

which is precisely (35)-(36). The last statement follows from the fact that $\operatorname{argmin} \Phi_{\lambda}=\operatorname{argmin} \Phi$ for all $\lambda>0$.

5.3. Rate of convergence of the values. Let $x:\left[t_{0},+\infty\left[\rightarrow \mathcal{H}\right.\right.$ be a solution of (RIGS) ${ }_{\gamma, \lambda}$. Let us fix $\bar{x} \in \operatorname{argmin} \Phi$, and set $h=h_{\bar{x}}$, that is, $h:\left[t_{0},+\infty\left[\rightarrow \mathbb{R}_{+}\right.\right.$satisfies $h(t)=\frac{1}{2}\|x(t)-\bar{x}\|^{2}$. We define the function $p:\left[t_{0},+\infty\left[\rightarrow \mathbb{R}_{+}\right.\right.$by $p(t)=e^{\int_{t_{0}}^{t} \gamma(\tau) d \tau}$. Under the assumption

$$
\left(H_{0}\right) \quad \int_{t_{0}}^{+\infty} \frac{d s}{p(s)}<+\infty,
$$

the function $\Gamma:\left[t_{0},+\infty\left[\rightarrow \mathbb{R}_{+}\right.\right.$is defined by $\Gamma(t)=p(t) \int_{t}^{+\infty} \frac{d s}{p(s)}$. Clearly, the function $\Gamma$ is of class $\mathcal{C}^{1}$ and satisfies

$$
\dot{\Gamma}(t)=\gamma(t) \Gamma(t)-1, \quad t \geq t_{0} .
$$

Let us define the function $\mathcal{E}:\left[t_{0},+\infty[\rightarrow \mathbb{R}\right.$ by

$$
\begin{aligned}
\mathcal{E}(t) & =\Gamma(t)^{2} W(t)+h(t)+\Gamma(t) \dot{h}(t) \\
& =\Gamma(t)^{2}\left(\frac{1}{2}\|\dot{x}(t)\|^{2}+\Phi_{\lambda(t)}(x(t))-\min _{\mathcal{H}} \Phi\right)+\frac{1}{2}\|x(t)-\bar{x}\|^{2}+\Gamma(t)\langle\dot{x}(t), x(t)-\bar{x}\rangle \\
& =\Gamma(t)^{2}\left(\Phi_{\lambda(t)}(x(t))-\min _{\mathcal{H}} \Phi\right)+\frac{1}{2}\|x(t)-\bar{x}+\Gamma(t) \dot{x}(t)\|^{2} .
\end{aligned}
$$

The following rate of convergence analysis is based on the decreasing properties of the function $\mathcal{E}$, that will serve us as a Lyapunov function.

Proposition 5.3 (Decay of $\mathcal{E}$ ). Let $\gamma:\left[t_{0},+\infty\left[\rightarrow \mathbb{R}_{+}\right.\right.$be a continuous function satisfying $\left(H_{0}\right)$. The energy function $\mathcal{E}:\left[t_{0},+\infty\left[\rightarrow \mathbb{R}_{+}\right.\right.$satisfies for every $t \geq t_{0}$,

$$
\dot{\mathcal{E}}(t)+\Gamma(t)(3-2 \gamma(t) \Gamma(t))\left(\Phi_{\lambda(t)}(x(t))-\min _{\mathcal{H}} \Phi\right) \leq 0 .
$$

Under the assumption

$$
\text { There exists } t_{1} \geq t_{0} \text { such that } \gamma(t) \Gamma(t) \leq 3 / 2 \text { for every } t \geq t_{1} \text {, }
$$

then we have $\dot{\mathcal{E}}(t) \leq 0$ for every $t \geq t_{1}$. 
Proof. By differentiating the function $\mathcal{E}$, as expressed in (38), we obtain

$$
\dot{\mathcal{E}}(t)=\Gamma(t)^{2} \dot{W}(t)+2 \Gamma(t) \dot{\Gamma}(t) W(t)+(1+\dot{\Gamma}(t)) \dot{h}(t)+\Gamma(t) \ddot{h}(t) .
$$

Taking into account the expression of $W$ and $\dot{W}$, along with equalities (35) and (37), we obtain

$$
\begin{aligned}
\dot{\mathcal{E}}(t)= & \Gamma(t)^{2} \dot{W}(t)+2 \Gamma(t) \dot{\Gamma}(t) W(t)+\Gamma(t)(\ddot{h}(t)+\gamma(t) \dot{h}(t)) \\
= & -\Gamma(t)^{2}\left(\gamma(t)\|\dot{x}(t)\|^{2}+\frac{\dot{\lambda}(t)}{2}\left\|\nabla \Phi_{\lambda(t)}(x(t))\right\|^{2}\right)+2 \Gamma(t) \dot{\Gamma}(t)\left(\frac{1}{2}\|\dot{x}(t)\|^{2}+\Phi_{\lambda(t)}(x(t))-\min _{\mathcal{H}} \Phi\right) \\
& +\Gamma(t)\left(\|\dot{x}(t)\|^{2}-\left\langle\nabla \Phi_{\lambda(t)}(x(t)), x(t)-\bar{x}\right\rangle\right) \\
\leq & \|\dot{x}(t)\|^{2} \Gamma(t)(-\Gamma(t) \gamma(t)+\dot{\Gamma}(t)+1)+2 \Gamma(t) \dot{\Gamma}(t)\left(\Phi_{\lambda(t)}(x(t))-\min _{\mathcal{H}} \Phi\right) \\
& -\Gamma(t)\left\langle\nabla \Phi_{\lambda(t)}(x(t)), x(t)-\bar{x}\right\rangle \\
= & 2 \Gamma(t) \dot{\Gamma}(t)\left(\Phi_{\lambda(t)}(x(t))-\min _{\mathcal{H}} \Phi\right)-\Gamma(t)\left\langle\nabla \Phi_{\lambda(t)}(x(t)), x(t)-\bar{x}\right\rangle .
\end{aligned}
$$

In the above calculation, we have neglected the term $-\Gamma(t)^{2} \frac{\dot{\lambda}(t)}{2}\left\|\nabla \Phi_{\lambda(t)}(x(t))\right\|^{2}$ which is less or equal than zero, because $\lambda(\cdot)$ is a nondecreasing function. To obtain the last equality, we have used again the equality $-\Gamma(t) \gamma(t)+\dot{\Gamma}(t)+1=0$. Let us now use the convexity of $\Phi_{\lambda(t)}$ and equality (37) to obtain

$$
\begin{aligned}
\dot{\mathcal{E}}(t) & \leq-(\Gamma(t)-2 \Gamma(t) \dot{\Gamma}(t))\left(\Phi_{\lambda(t)}(x(t))-\min _{\mathcal{H}} \Phi\right) \\
& =-\Gamma(t)(3-2 \gamma(t) \Gamma(t))\left(\Phi_{\lambda(t)}(x(t))-\min _{\mathcal{H}} \Phi\right) .
\end{aligned}
$$

When $\left(K_{1}\right)$ is satisfied, we have $3-2 \gamma(t) \Gamma(t) \geq 0$. Since $\Gamma(t)$ and $\Phi_{\lambda(t)}(x(t))-\min _{\mathcal{H}} \Phi$ are nonnegative, we deduce that $\dot{\mathcal{E}}(t) \leq 0$.

Corollary 5.4. Let $\gamma:\left[t_{0},+\infty\left[\rightarrow \mathbb{R}_{+}\right.\right.$be a continuous function satisfying $\left(H_{0}\right)$ and $\left(K_{1}\right)$.

(i) For every $t \geq t_{1}$, we have

$$
\Phi_{\lambda(t)}(x(t))-\min _{\mathcal{H}} \Phi \leq \frac{\mathcal{E}\left(t_{1}\right)}{\Gamma(t)^{2}} .
$$

As a consequence, setting $\xi(t)=\operatorname{prox}_{\lambda(t) \Phi}(x(t))$, we have

$$
\Phi(\xi(t))-\min \Phi \leq \frac{\mathcal{E}\left(t_{1}\right)}{\Gamma(t)^{2}} \quad \text { and } \quad\|x(t)-\xi(t)\|^{2} \leq \frac{2 \lambda(t)}{\Gamma(t)^{2}} \mathcal{E}\left(t_{1}\right) .
$$

(ii) Assume moreover that

$\left(K_{1}^{+}\right) \quad$ There exist $t_{1} \geq t_{0}$ and $m<3 / 2$ such that $\gamma(t) \Gamma(t) \leq m$ for every $t \geq t_{1}$.

Then we have

$$
\int_{t_{1}}^{+\infty} \Gamma(t)\left(\Phi_{\lambda(t)}(x(t))-\min _{\mathcal{H}} \Phi\right) d t \leq \frac{\mathcal{E}\left(t_{1}\right)}{3-2 m}<+\infty .
$$

Proof. (i) From Proposition 5.3, the function $\mathcal{E}$ is nonincreasing on $\left[t_{1},+\infty\left[\right.\right.$. It ensues that $\mathcal{E}(t) \leq \mathcal{E}\left(t_{1}\right)$ for every $t \geq t_{1}$. Taking into account the expression (39), we deduce that for every $t \geq t_{1}$,

$$
\Gamma(t)^{2}\left(\Phi_{\lambda(t)}(x(t))-\min _{\mathcal{H}} \Phi\right) \leq \mathcal{E}\left(t_{1}\right) \quad \text { and } \quad \frac{1}{2}\|x(t)-\bar{x}+\Gamma(t) \dot{x}(t)\|^{2} \leq \mathcal{E}\left(t_{1}\right) .
$$

The first assertion follows immediately.

(ii) Now assume $\left(K_{1}^{+}\right)$. By integrating (40) on $\left[t_{1}, t\right]$, we find

$$
\mathcal{E}(t)+\int_{t_{1}}^{t}\left(\Phi_{\lambda(s)}(x(s))-\min _{\mathcal{H}} \Phi\right) \Gamma(s)(3-2 \gamma(s) \Gamma(s)) d s \leq \mathcal{E}\left(t_{1}\right) .
$$


Since $\mathcal{E}(t) \geq 0$ and $\gamma(t) \Gamma(t) \leq m$ for every $t \geq t_{1}$, this implies that

$$
(3-2 m) \int_{t_{1}}^{t} \Gamma(s)\left(\Phi_{\lambda(s)}(x(s))-\min _{\mathcal{H}} \Phi\right) d s \leq \mathcal{E}\left(t_{1}\right) .
$$

The inequality (41) is obtained by letting $t$ tend toward infinity.

Proposition 5.5. Let $\gamma:\left[t_{0},+\infty\left[\rightarrow \mathbb{R}_{+}\right.\right.$be a continuous function satisfying $\left(H_{0}\right)$ and $\left(K_{1}^{+}\right)$. Then, we have

(i) $\int_{t_{0}}^{+\infty} \Gamma(t)\|\dot{x}(t)\|^{2} d t<+\infty$, and hence $\int_{t_{0}}^{+\infty} \Gamma(t) W(t) d t<+\infty$;

(ii) $\int_{t_{0}}^{+\infty} \gamma(t)\left(\int_{t_{0}}^{t} \Gamma(s) d s\right)\|\dot{x}(t)\|^{2} d t<+\infty$.

Proof. By (34) and $\lambda$ nondecreasing we have

$$
\dot{W}(t) \leq-\gamma(t)\|\dot{x}(t)\|^{2} .
$$

Let $\theta:\left[t_{0},+\infty\left[\rightarrow \mathbb{R}_{+}\right.\right.$be a differentiable test function, and let $t_{1} \geq t_{0}$ be given by the assumption $\left(K_{1}^{+}\right)$. Let us multiply the inequality (42) by $\theta(t)$ and integrate on $\left[t_{1}, t\right]$

$$
\int_{t_{1}}^{t} \theta(s) \dot{W}(s) d s+\int_{t_{1}}^{t} \theta(s) \gamma(s)\|\dot{x}(s)\|^{2} d s \leq 0 .
$$

Integrating by parts yields

$$
\theta(t) W(t)+\int_{t_{1}}^{t} \theta(s) \gamma(s)\|\dot{x}(s)\|^{2} d s \leq \theta\left(t_{1}\right) W\left(t_{1}\right)+\int_{t_{1}}^{t} \dot{\theta}(s) W(s) d s .
$$

Using the expression of $W$ and rearranging the terms, we find

$$
\theta(t) W(t)+\int_{t_{1}}^{t}(\theta(s) \gamma(s)-\dot{\theta}(s) / 2)\|\dot{x}(s)\|^{2} d s \leq \theta\left(t_{1}\right) W\left(t_{1}\right)+\int_{t_{1}}^{t} \dot{\theta}(s)\left(\Phi_{\lambda(s)}(x(s))-\min _{\mathcal{H}} \Phi\right) d s .
$$

(i) Choosing $\theta(t)=\Gamma(t)^{2}$, the above equality gives for every $t \geq t_{1}$,

$\Gamma(t)^{2} W(t)+\int_{t_{1}}^{t} \Gamma(s)[\Gamma(s) \gamma(s)-\dot{\Gamma}(s)]\|\dot{x}(s)\|^{2} d s \leq \Gamma\left(t_{1}\right)^{2} W\left(t_{1}\right)+2 \int_{t_{1}}^{t} \Gamma(s) \dot{\Gamma}(s)\left(\Phi_{\lambda(s)}(x(s))-\min _{\mathcal{H}} \Phi\right) d s$.

Recalling that $\dot{\Gamma}=\gamma \Gamma-1$, we deduce that

$$
\Gamma(t)^{2} W(t)+\int_{t_{1}}^{t} \Gamma(s)\|\dot{x}(s)\|^{2} d s \leq \Gamma\left(t_{1}\right)^{2} W\left(t_{1}\right)+2 \int_{t_{1}}^{t} \Gamma(s)(\gamma(s) \Gamma(s)-1)\left(\Phi_{\lambda(s)}(x(s))-\min _{\mathcal{H}} \Phi\right) d s .
$$

By assumption $\left(K_{1}^{+}\right)$, we have $\gamma(t) \Gamma(t) \leq 3 / 2$ for every $t \geq t_{1}$. Since $W(t) \geq 0$, it ensues that

$$
\int_{t_{1}}^{t} \Gamma(s)\|\dot{x}(s)\|^{2} d s \leq \Gamma\left(t_{1}\right)^{2} W\left(t_{1}\right)+\int_{t_{1}}^{t} \Gamma(s)\left(\Phi_{\lambda(s)}(x(s))-\min _{\mathcal{H}} \Phi\right) d s .
$$

$\operatorname{Under}\left(K_{1}^{+}\right)$, we have the estimate $\int_{t_{1}}^{+\infty} \Gamma(s)\left(\Phi_{\lambda(s)}(x(s))-\min _{\mathcal{H}} \Phi\right) d s<+\infty$, see Corollary $5.4(i i)$. The announced estimates follow immediately.

(ii) Take now $\theta(t)=\int_{t_{0}}^{t} \Gamma(s) d s$. Recalling that $W(t) \geq 0$, inequality (43) then implies that for every $t \geq t_{1}$

$$
\int_{t_{1}}^{t}\left[\gamma(s)\left(\int_{t_{0}}^{s} \Gamma(u) d u\right)\right]\|\dot{x}(s)\|^{2} d s \leq\left(\int_{t_{0}}^{t_{1}} \Gamma(s) d s\right) W\left(t_{1}\right)+\int_{t_{1}}^{t} \Gamma(s) W(s) d s .
$$

It suffices then to recall that $\int_{t_{1}}^{+\infty} \Gamma(s) W(s) d s<+\infty$ under hypothesis $\left(K_{1}^{+}\right)$, see point $(i)$. 
Theorem 5.6. Let $\gamma:\left[t_{0},+\infty\left[\rightarrow \mathbb{R}_{+}\right.\right.$be a continuous function satisfying $\left(H_{0}\right),\left(K_{1}^{+}\right)$, along with

$$
\int_{t_{0}}^{+\infty} \Gamma(s) d s=+\infty
$$

Let $x($.$) be a solution of (RIGS) )_{\gamma, \lambda}$. Then we have

$$
\Phi_{\lambda(t)}(x(t))-\min _{\mathcal{H}} \Phi=o\left(\frac{1}{\int_{t_{0}}^{t} \Gamma(s) d s}\right) \quad \text { and } \quad\|\dot{x}(t)\|=o\left(\frac{1}{\int_{t_{0}}^{t} \Gamma(s) d s}\right)^{1 / 2} \text { as } t \rightarrow+\infty .
$$

As a consequence, setting $\xi(t)=\operatorname{prox}_{\lambda(t) \Phi}(x(t))$, we have

$$
\Phi(\xi(t))-\min _{\mathcal{H}} \Phi=o\left(\frac{1}{\int_{t_{0}}^{t} \Gamma(s) d s}\right) \quad \text { and } \quad\|x(t)-\xi(t)\|=o\left(\frac{\lambda(t)}{\int_{t_{0}}^{t} \Gamma(s) d s}\right)^{1 / 2} \quad \text { as } t \rightarrow+\infty .
$$

In particular, we obtain $\lim _{t \rightarrow+\infty} \Phi(\xi(t))=\min _{\mathcal{H}} \Phi$, and $\lim _{t \rightarrow+\infty}\|\dot{x}(t)\|=0$.

Proof. From Proposition $5.5(i)$, we have $\int_{t_{0}}^{+\infty} \Gamma(t) W(t) d t<+\infty$. On the other hand, the energy function $W$ is nonincreasing by Proposition 5.1. By applying Lemma B.3 in the Appendix, we obtain that

$$
W(t)=o\left(\frac{1}{\int_{t_{0}}^{t} \Gamma(s) d s}\right) \text { as } t \rightarrow+\infty .
$$

The announced estimates follow immediately.

Theorem 5.7. Let $\gamma:\left[t_{0},+\infty\left[\rightarrow \mathbb{R}_{+}\right.\right.$be a continuous function satisfying $\left(H_{0}\right),\left(K_{1}^{+}\right)$, and $\left(K_{2}\right)$. Suppose that $\lambda:\left[t_{0},+\infty\left[\rightarrow \mathbb{R}_{+}^{*}\right.\right.$ is nondecreasing and satisfies

$$
\sup _{t \geq t_{0}} \frac{\lambda(t)}{\int_{t_{0}}^{t} \Gamma(s) d s}<+\infty .
$$

Then, for every solution $x($.$) of (RIGS) { }_{\gamma, \lambda}$ the following properties hold:

(i) $\lim _{t \rightarrow+\infty}\|\xi(t)-x(t)\|=0, \quad$ where $\xi(t)=\operatorname{prox}_{\lambda(t) \Phi}(x(t))$;

(ii) $x(t)$ converges weakly as $t \rightarrow+\infty$ toward some $x^{*} \in \operatorname{argmin} \Phi$.

Proof. (i) Since $\sup _{t \geq t_{0}} \frac{\lambda(t)}{\int_{t_{0}}^{t} \Gamma(s) d s}<+\infty$, the second estimate of (44) implies that $\lim _{t \rightarrow+\infty}\|\xi(t)-x(t)\|=0$.

(ii) We apply the Opial lemma, see Lemma 2.2. Let us fix $\bar{x} \in \operatorname{argmin} \Phi$, and show that $\lim _{t \rightarrow+\infty}\|x(t)-\bar{x}\|$ exists. For that purpose, let us set $h(t)=\frac{1}{2}\|x(t)-\bar{x}\|^{2}$. Recall from Lemma 5.2 that the function $h$ satisfies the following differential inequality

$$
\ddot{h}(t)+\gamma(t) \dot{h}(t) \leq\|\dot{x}(t)\|^{2} .
$$

From Proposition $5.5(i)$, we have $\int_{t_{0}}^{+\infty} \Gamma(s)\|\dot{x}(s)\|^{2} d s<+\infty$. By applying Lemma B.1 with $g:\left[t_{0},+\infty[\rightarrow\right.$ $\mathbb{R}_{+}$defined by $g(t)=\|\dot{x}(t)\|^{2}$, we obtain that $\lim _{t \rightarrow+\infty} h(t)$ exists. This shows the first point of the Opial lemma. Let us now verify the second point. Let $x\left(t_{k}\right)$ converge weakly to $x_{\infty}$ as $k \rightarrow+\infty$. Point $(i)$ implies that $\xi\left(t_{k}\right)$ also converges weakly to $x_{\infty}$ as $k \rightarrow+\infty$. Since the function $\Phi$ is convex and lower semicontinuous, it is semicontinuous for the weak topology, hence satisfies

$$
\Phi\left(x_{\infty}\right) \leq \liminf _{t \rightarrow+\infty} \Phi\left(\xi\left(t_{k}\right)\right)=\lim _{t \rightarrow+\infty} \Phi(\xi(t))=\min _{\mathcal{H}} \Phi,
$$

$c f$. the last point of Theorem 5.6. It ensues that $x_{\infty} \in \operatorname{argmin} \Phi$, which establishes the second point of the Opial lemma, and ends the proof. 


\section{Appendix A. Yosida regularization and Moreau envelopes}

A.1. Yosida regularization of an operator $A$. A set-valued mapping $A$ from $\mathcal{H}$ to $\mathcal{H}$ assigns to each $x \in \mathcal{H}$ a set $A(x) \subset \mathcal{H}$, hence it is a mapping from $\mathcal{H}$ to $2^{\mathcal{H}}$. Every set-valued mappping $A: \mathcal{H} \rightarrow 2^{\mathcal{H}}$ can be identified with its graph defined by

$$
\operatorname{gph} A=\{(x, u) \in \mathcal{H} \times \mathcal{H}: u \in A(x)\} .
$$

The set $\{x \in \mathcal{H}: 0 \in A(x)\}$ of the zeros of $A$ is denoted by zer $A$. An operator $A: \mathcal{H} \rightarrow 2^{\mathcal{H}}$ is said to be monotone if for any $(x, u),(y, v) \in \operatorname{gph} A$, one has $\langle y-x, v-u\rangle \geq 0$. It is maximally monotone if there exists no monotone operator whose graph strictly contains gph $A$. If a single-valued operator $A: \mathcal{H} \rightarrow \mathcal{H}$ is continuous and monotone, then it is maximally monotone, $c f$. [15, Proposition 2.4].

Given a maximally monotone operator $A$ and $\lambda>0$, the resolvent of $A$ with index $\lambda$ and the Yosida regularization of $A$ with parameter $\lambda$ are defined by

$$
J_{\lambda A}=(I+\lambda A)^{-1} \quad \text { and } \quad A_{\lambda}=\frac{1}{\lambda}\left(I-J_{\lambda A}\right),
$$

respectively. The operator $J_{\lambda A}: \mathcal{H} \rightarrow \mathcal{H}$ is nonexpansive and eveywhere defined (indeed it is firmly non-expansive). Moreover, $A_{\lambda}$ is $\lambda$-cocoercive: for all $x, y \in \mathcal{H}$ we have

$$
\left\langle A_{\lambda} y-A_{\lambda} x, y-x\right\rangle \geq \lambda\left\|A_{\lambda} y-A_{\lambda} x\right\|^{2} .
$$

This property immediately implies that $A_{\lambda}: \mathcal{H} \rightarrow \mathcal{H}$ is $\frac{1}{\lambda}$-Lipschitz continuous. Another property that proves useful is the resolvent equation (see, for example, [15, Proposition 2.6] or [11, Proposition 23.6])

$$
\left(A_{\lambda}\right)_{\mu}=A_{(\lambda+\mu)},
$$

which is valid for any $\lambda, \mu>0$. This property allows to compute simply the resolvent of $A_{\lambda}$ by

$$
J_{\mu A_{\lambda}}=\frac{\lambda}{\lambda+\mu} I+\frac{\mu}{\lambda+\mu} J_{(\lambda+\mu) A},
$$

for any $\lambda, \mu>0$. Also note that for any $x \in \mathcal{H}$, and any $\lambda>0$

$$
A_{\lambda}(x) \in A\left(J_{\lambda A} x\right)=A\left(x-\lambda A_{\lambda}(x)\right) .
$$

Finally, for any $\lambda>0, A$ and $A_{\lambda}$ have the same solution set $S:=A_{\lambda}^{-1}(0)=A^{-1}(0)$. For a detailed presentation of the properties of the maximally monotone operators and the Yosida approximation, the reader can consult [11] or [15].

\section{A.2. Differentiability properties of the Moreau envelopes.}

Lemma A.1. For each $x \in \mathcal{H}$, the real-valued function $\lambda \mapsto \Phi_{\lambda}(x)$ is continuously differentiable on ] $0,+\infty[$, with

$$
\frac{d}{d \lambda} \Phi_{\lambda}(x)=-\frac{1}{2}\left\|\nabla \Phi_{\lambda}(x)\right\|^{2}
$$

Proof. By definition of $\Phi_{\lambda}$, we have

$$
\lambda \Phi_{\lambda}(x)=\inf _{\xi \in \mathcal{H}}\left\{\lambda \Phi(\xi)+\frac{1}{2}\|x-\xi\|^{2}\right\}=\lambda \Phi\left(J_{\lambda}(x)\right)+\frac{1}{2}\left\|x-J_{\lambda}(x)\right\|^{2},
$$

where the infimum in the above expression is achieved at $J_{\lambda}(x):=(I+\lambda \partial \Phi)^{-1}(x)$. Let us prove that

$$
\frac{d}{d \lambda} \lambda \Phi_{\lambda}(x)=\Phi\left(J_{\lambda}(x)\right)
$$


This will immediately give our result, since, by the derivation chain rule,

$$
\begin{aligned}
\frac{d}{d \lambda} \Phi_{\lambda}(x) & =\frac{d}{d \lambda}\left(\frac{1}{\lambda} \times \lambda \Phi_{\lambda}(x)\right)=\frac{1}{\lambda} \Phi\left(J_{\lambda}(x)\right)-\frac{1}{\lambda^{2}} \lambda \Phi_{\lambda}(x) \\
& =-\frac{1}{\lambda}\left(\Phi_{\lambda}(x)-\Phi\left(J_{\lambda}(x)\right)\right) \\
& =-\frac{1}{2 \lambda^{2}}\left\|x-J_{\lambda}(x)\right\|^{2} \\
& =-\frac{1}{2}\left\|\nabla \Phi_{\lambda}(x)\right\|^{2}
\end{aligned}
$$

To obtain (47), take two values $\lambda_{1}$ and $\lambda_{2}$ of the parameter $\lambda$, and compare the corresponding values of the function $\lambda \mapsto \lambda \Phi_{\lambda}(x)$. By the formulation (46) of $\lambda \Phi_{\lambda}(x)$ as an infimal value, we have

$$
\begin{aligned}
\lambda_{1} \Phi_{\lambda_{1}}(x)-\lambda_{2} \Phi_{\lambda_{2}}(x) & \leq \lambda_{1} \Phi\left(J_{\lambda_{2}}(x)\right)+\frac{1}{2}\left\|x-J_{\lambda_{2}}(x)\right\|^{2}-\lambda_{2} \Phi\left(J_{\lambda_{2}}(x)\right)-\frac{1}{2}\left\|x-J_{\lambda_{2}}(x)\right\|^{2} \\
& =\left(\lambda_{1}-\lambda_{2}\right) \Phi\left(J_{\lambda_{2}}(x)\right) .
\end{aligned}
$$

Exchanging the roles of $\lambda_{1}$ and $\lambda_{2}$, we obtain

$$
\left(\lambda_{1}-\lambda_{2}\right) \Phi\left(J_{\lambda_{1}}(x)\right) \leq \lambda_{1} \Phi_{\lambda_{1}}(x)-\lambda_{2} \Phi_{\lambda_{2}}(x) \leq\left(\lambda_{1}-\lambda_{2}\right) \Phi\left(J_{\lambda_{2}}(x)\right) .
$$

Then note that the mapping $\lambda \mapsto \Phi\left(J_{\lambda}(x)\right)$ is continuous. This follows from (46) and the continuity of the mappings $\lambda \mapsto \Phi_{\lambda}(x)$ and $\lambda \mapsto J_{\lambda}(x)$. Indeed, these mappings are locally Lipschitz continuous. This is a direct consequence of the resolvent equations (33), see [11, Proposition 23.28] for further details. Then divide the above formula by $\lambda_{1}-\lambda_{2}$ (successively examining the two cases $\lambda_{1}<\lambda_{2}$, then $\lambda_{2}<\lambda_{1}$ ). Letting $\lambda_{1} \rightarrow \lambda_{2}$, and using the continuity of $\lambda \mapsto \Phi\left(J_{\lambda}(x)\right)$ gives the differentiability of the mapping $\lambda \mapsto \lambda \Phi_{\lambda}(x)$, and formula (47). Then, writing $\Phi_{\lambda}(x)=\frac{1}{\lambda}\left(\lambda \Phi_{\lambda}(x)\right)$, and applying the derivation chain rule gives (45). The continuity of $\lambda \mapsto \nabla \Phi_{\lambda}(x)$ gives the continuous differentiability of $\lambda \mapsto \Phi_{\lambda}(x)$.

\section{Appendix B. Some AuXiLiary RESUlts}

In this section, we present some auxiliary lemmas that are used throughout the paper. The following result allows us to establish some majorization and also the convergence as $t \rightarrow+\infty$ of a real-valued function satisfying some differential inequality.

Lemma B.1. Let $\gamma:\left[t_{0},+\infty\left[\rightarrow \mathbb{R}_{+}\right.\right.$be a continuous function satisfying $\int_{t_{0}}^{+\infty} \frac{d s}{p(s)}<+\infty$, where the function $p$ is defined by $p(t)=e^{\int_{t_{0}}^{t} \gamma(\tau) d \tau}$. Let $g:\left[t_{0},+\infty[\rightarrow \mathbb{R}\right.$ be a continuous function. Assume that $h:\left[t_{0},+\infty\left[\rightarrow \mathbb{R}_{+}\right.\right.$is a function of class $\mathcal{C}^{2}$ satisfying

$$
\ddot{h}(t)+\gamma(t) \dot{h}(t) \leq g(t) \quad \text { on }\left[t_{0},+\infty[.\right.
$$

(i) For every $t \geq t_{0}$, we have

$$
h(t) \leq h\left(t_{0}\right)+\dot{h}\left(t_{0}\right)\left(\int_{t_{0}}^{t} \frac{d u}{p(u)}\right)+\int_{t_{0}}^{t}\left(\int_{s}^{t} \frac{d u}{p(u)}\right) p(s) g(s) d s .
$$

(ii) Assume that $\int_{t_{0}}^{+\infty} \Gamma(s)|g(s)| d s<+\infty$, where $\Gamma:\left[t_{0},+\infty\left[\rightarrow \mathbb{R}_{+}\right.\right.$is given by $\Gamma(t)=p(t) \int_{t}^{+\infty} \frac{d s}{p(s)}$. Then the nonnegative part $\dot{h}_{+}$of $\dot{h}$ belongs to $L^{1}\left(t_{0},+\infty\right)$, and hence $\lim _{t \rightarrow+\infty} h(t)$ exists.

Proof. ( $i$ ) Let us multiply each member of inequality (48) by $p(t)=e^{\int_{t_{0}}^{t} \gamma(\tau) d \tau}$ and integrate on $\left[t_{0}, t\right]$. We obtain

$$
\dot{h}(t) \leq \dot{h}\left(t_{0}\right) \frac{1}{p(t)}+\frac{1}{p(t)} \int_{t_{0}}^{t} p(s) g(s) d s
$$


By integrating again on $\left[t_{0}, t\right]$, we find

$$
h(t) \leq h\left(t_{0}\right)+\dot{h}\left(t_{0}\right)\left(\int_{t_{0}}^{t} \frac{d u}{p(u)}\right)+\int_{t_{0}}^{t} \frac{1}{p(u)}\left(\int_{t_{0}}^{u} p(s) g(s) d s\right) d u .
$$

From Fubini theorem, we have

$$
\int_{t_{0}}^{t} \frac{1}{p(u)}\left(\int_{t_{0}}^{u} p(s) g(s) d s\right) d u=\int_{t_{0}}^{t}\left(\int_{s}^{t} \frac{d u}{p(u)}\right) p(s) g(s) d s,
$$

and the inequality (49) follows immediately.

(ii) Let us now assume that $\int_{t_{0}}^{+\infty} \Gamma(s)|g(s)| d s<+\infty$. We easily deduce from (50) that for every $t \geq t_{0}$,

$$
\dot{h}_{+}(t) \leq\left|\dot{h}\left(t_{0}\right)\right| \frac{1}{p(t)}+\frac{1}{p(t)} \int_{t_{0}}^{t} p(s)|g(s)| d s .
$$

By applying Fubini theorem, we find

$$
\begin{aligned}
\int_{t_{0}}^{+\infty} \frac{1}{p(t)}\left(\int_{t_{0}}^{t} p(s)|g(s)| d s\right) d t & =\int_{t_{0}}^{+\infty}\left(\int_{s}^{+\infty} \frac{d t}{p(t)}\right) p(s)|g(s)| d s \\
& =\int_{t_{0}}^{+\infty} \Gamma(s)|g(s)| d s<+\infty
\end{aligned}
$$

Since $\int_{t_{0}}^{+\infty} \frac{d t}{p(t)}<+\infty$ by assumption, we deduce from (51) and (52) that $\dot{h}_{+} \in L^{1}\left(t_{0},+\infty\right)$. Hence $\lim _{t \rightarrow+\infty} h(t)$ exists.

Let us now state a vector-valued version of Lemma B.1.

Lemma B.2. Let $\gamma:\left[t_{0},+\infty\left[\rightarrow \mathbb{R}_{+}\right.\right.$be a continuous function satisfying $\int_{t_{0}}^{+\infty} \frac{d s}{p(s)}<+\infty$, where the function $p$ is defined by $p(t)=e^{\int_{t_{0}}^{t} \gamma(\tau) d \tau}$. Let $F:\left[t_{0},+\infty[\rightarrow \mathcal{H}\right.$ be a measurable map such that $\int_{t_{0}}^{+\infty} \Gamma(t)\|F(t)\| d t<+\infty$. Assume that $x:\left[t_{0},+\infty\left[\rightarrow \mathcal{H}\right.\right.$ is a map of class $\mathcal{C}^{2}$ satisfying

$$
\ddot{x}(t)+\gamma(t) \dot{x}(t)=F(t) \quad \text { on }\left[t_{0},+\infty[\text {. }\right.
$$

Then $\|\dot{x}\| \in L^{1}\left(t_{0},+\infty\right)$, and hence $x(t)$ converges strongly as $t \rightarrow+\infty$.

Proof. Let us multiply (53) by $p(t)=e^{\int_{t_{0}}^{t} \gamma(\tau) d \tau}$ and integrate on $\left[t_{0}, t\right]$. We obtain for every $t \geq t_{0}$,

$$
\dot{x}(t)=\dot{x}\left(t_{0}\right) \frac{1}{p(t)}+\frac{1}{p(t)} \int_{t_{0}}^{t} p(s) F(s) d s .
$$

Taking the norm of each member, we deduce that

$$
\|\dot{x}(t)\| \leq\left\|\dot{x}\left(t_{0}\right)\right\| \frac{1}{p(t)}+\frac{1}{p(t)} \int_{t_{0}}^{t} p(s)\|F(s)\| d s .
$$

By integrating and applying Fubini theorem as in the proof of Lemma B.1, we find

$$
\int_{t_{0}}^{+\infty}\|\dot{x}(t)\| d t \leq\left\|\dot{x}\left(t_{0}\right)\right\| \int_{t_{0}}^{+\infty} \frac{d t}{p(t)}+\int_{t_{0}}^{+\infty} \Gamma(s)\|F(s)\| d s<+\infty .
$$

The strong convergence of $x(t)$ as $t \rightarrow+\infty$ follows immediately.

Owing to the next lemma, we can estimate the rate of convergence of a function $w:\left[t_{0},+\infty\left[\rightarrow \mathbb{R}_{+}\right.\right.$ supposed to be nonincreasing and summable with respect to a weight function $\Gamma$. 
Lemma B.3. Let $\Gamma:\left[t_{0},+\infty\left[\rightarrow \mathbb{R}_{+}\right.\right.$be a measurable function such that $\int_{t_{0}}^{+\infty} \Gamma(t) d t=+\infty$. Assume that $w:\left[t_{0},+\infty\left[\rightarrow \mathbb{R}_{+}\right.\right.$is nonincreasing and satisfies $\int_{t_{0}}^{+\infty} \Gamma(t) w(t) d t<+\infty$. Then we have

$$
w(t)=o\left(\frac{1}{\int_{t_{0}}^{t} \Gamma(s) d s}\right) \quad \text { as } t \rightarrow+\infty .
$$

Proof. Let $F:\left[t_{0},+\infty\left[\rightarrow \mathbb{R}_{+}\right.\right.$be the function defined by $F(t)=\int_{t_{0}}^{t} \Gamma(s) d s$. It follows from the hypothesis $\int_{t_{0}}^{+\infty} \Gamma(s) d s=+\infty$ that the function $F$ is an increasing bijection from $\left[t_{0},+\infty[\right.$ onto $[0,+\infty[$. For every $t \geq t_{0}$, let us set $\alpha(t)=F^{-1}\left(\frac{1}{2} F(t)\right)$. By definition, we have $\int_{t_{0}}^{\alpha(t)} \Gamma(s) d s=\frac{1}{2} \int_{t_{0}}^{t} \Gamma(s) d s$, hence $\int_{\alpha(t)}^{t} \Gamma(s) d s=\frac{1}{2} \int_{t_{0}}^{t} \Gamma(s) d s$. Recalling that the function $w$ is nonincreasing, we obtain

$$
\int_{\alpha(t)}^{t} \Gamma(s) w(s) d s \geq w(t) \int_{\alpha(t)}^{t} \Gamma(s) d s=\frac{1}{2} w(t) \int_{t_{0}}^{t} \Gamma(s) d s .
$$

By assumption, we have $\int_{t_{0}}^{+\infty} \Gamma(s) w(s) d s<+\infty$. Since $\lim _{t \rightarrow+\infty} \alpha(t)=+\infty$, we deduce that

$$
\lim _{t \rightarrow+\infty} \int_{\alpha(t)}^{t} \Gamma(s) w(s) d s=0 .
$$

The conclusion follows from the two above relations.

Given a Banach space $(\mathcal{X},\|\|$.$) and a bounded map x:\left[t_{0},+\infty[\rightarrow \mathcal{X}\right.$, the next lemma gives basic properties of the averaged trajectory $\widehat{x}$ defined by $(21)$.

Lemma B.4. Let us give $(\mathcal{X},\|\|$.$) a Banach space, \Lambda:\left[t_{0},+\infty\left[\times\left[t_{0},+\infty\left[\rightarrow \mathbb{R}_{+}\right.\right.\right.\right.$a measurable function satisfying (19), and $x:\left[t_{0},+\infty[\rightarrow \mathcal{X}\right.$ a bounded map. Then we have

(i) For every $t \geq t_{0}$, the vector $\widehat{x}(t)=\int_{t_{0}}^{+\infty} \Lambda(s, t) x(s) d s$ is well-defined. The map $\widehat{x}$ is bounded and $\sup _{t \geq t_{0}}\|\widehat{x}(t)\| \leq \sup _{t \geq t_{0}}\|x(t)\|$.

(ii) Assume moreover that the function $\Lambda$ satisfies (20). If $\lim _{t \rightarrow+\infty} x(t)=x_{\infty}$ for some $x_{\infty} \in \mathcal{X}$, then $\lim _{t \rightarrow+\infty} \widehat{x}(t)=x_{\infty}$.

Proof. (i) Let us set $M=\sup _{t \geq t_{0}}\|x(t)\|<+\infty$. In view of (19), observe that for every $t \geq t_{0}$,

$$
\int_{t_{0}}^{+\infty} \Lambda(s, t)\|x(s)\| d s \leq M \int_{t_{0}}^{+\infty} \Lambda(s, t) d s=M .
$$

Since $\mathcal{X}$ is complete, we classically deduce that the integral $\int_{t_{0}}^{+\infty} \Lambda(s, t) x(s) d s$ is convergent. From the definition of $\widehat{x}(t)$, we then have $\|\widehat{x}(t)\| \leq \int_{t_{0}}^{+\infty} \Lambda(s, t)\|x(s)\| d s$, and hence $\|\widehat{x}(t)\| \leq M$ in view of (54).

(ii) Assume that $\lim _{t \rightarrow+\infty} x(t)=x_{\infty}$ for some $x_{\infty} \in \mathcal{X}$. Observe that for every $t \geq t_{0}$,

$$
\begin{aligned}
\left\|\widehat{x}(t)-x_{\infty}\right\| & =\left\|\int_{t_{0}}^{+\infty} \Lambda(s, t)\left(x(s)-x_{\infty}\right) d s\right\| \quad \text { by using (19) } \\
& \leq \int_{t_{0}}^{+\infty} \Lambda(s, t)\left\|x(s)-x_{\infty}\right\| d s .
\end{aligned}
$$

Fix $\varepsilon>0$ and let $T \geq t_{0}$ be such that $\left\|x(t)-x_{\infty}\right\| \leq \varepsilon$ for every $t \geq T$. From (55), we obtain

$$
\begin{aligned}
\left\|\widehat{x}(t)-x_{\infty}\right\| & \leq\left(\sup _{t \in\left[t_{0}, T\right]}\left\|x(t)-x_{\infty}\right\|\right)\left(\int_{t_{0}}^{T} \Lambda(s, t) d s\right)+\varepsilon \int_{T}^{+\infty} \Lambda(s, t) d s \\
& \leq M \int_{t_{0}}^{T} \Lambda(s, t) d s+\varepsilon
\end{aligned}
$$


with $M=\sup _{t \geq t_{0}}\left\|x(t)-x_{\infty}\right\|<+\infty$. Taking the upper limit as $t \rightarrow+\infty$, we deduce from property (20) that

$$
\limsup _{t \rightarrow+\infty}\left\|\widehat{x}(t)-x_{\infty}\right\| \leq \varepsilon
$$

Since this is true for every $\varepsilon>0$, we conclude that $\lim _{t \rightarrow+\infty}\left\|\widehat{x}(t)-x_{\infty}\right\|=0$.

Lemma B.5. Let $(\mathcal{X},\|\|$.$) be a Banach space, and let x:\left[t_{0},+\infty[\rightarrow \mathcal{X}\right.$ be a continuous map, supposed to be bounded on $\left[t_{0},+\infty\left[\right.\right.$. Let $\Lambda_{1}, \Lambda_{2}:\left[t_{0},+\infty\left[\times\left[t_{0},+\infty\left[\rightarrow \mathbb{R}_{+}\right.\right.\right.\right.$be measurable functions satisfying (19). Assume that

$$
\lim _{t \rightarrow+\infty} \int_{t_{0}}^{+\infty}\left|\Lambda_{1}(s, t)-\Lambda_{2}(s, t)\right| d s=0 .
$$

Let us consider the averaged trajectories $\widehat{x}_{1}, \widehat{x}_{2}:\left[t_{0},+\infty[\rightarrow \mathcal{X}\right.$ defined by

$$
\widehat{x}_{1}(t)=\int_{t_{0}}^{+\infty} \Lambda_{1}(s, t) x(s) d s \quad \text { and } \quad \widehat{x}_{2}(t)=\int_{t_{0}}^{+\infty} \Lambda_{2}(s, t) x(s) d s .
$$

Then we have $\lim _{t \rightarrow+\infty}\left\|\widehat{x}_{1}(t)-\widehat{x}_{2}(t)\right\|=0$.

Proof. Let $M \geq 0$ be such that $\|x(t)\| \leq M$ for every $t \geq t_{0}$. Observe that

$$
\begin{aligned}
\left\|\widehat{x}_{1}(t)-\widehat{x}_{2}(t)\right\| & =\left\|\int_{t_{0}}^{+\infty}\left(\Lambda_{1}(s, t)-\Lambda_{2}(s, t)\right) x(s) d s\right\| \\
& \leq \int_{t_{0}}^{+\infty}\left|\Lambda_{1}(s, t)-\Lambda_{2}(s, t)\right|\|x(s)\| d s \\
& \left.\leq M \int_{t_{0}}^{+\infty} \mid \Lambda_{1}(s, t)-\Lambda_{2}(s, t)\right) \mid d s \longrightarrow 0 \quad \text { as } t \rightarrow+\infty, \text { in view of }(56) .
\end{aligned}
$$

\section{REFERENCES}

[1] F. Álvarez, H. Atтouch, The heavy ball with friction dynamical system for convex constrained minimization problems, Optimization (Namur, 1998), Lecture Notes in Econom. and Math. Systems 481, Springer, Berlin, (2000), pp. $25-35$.

[2] H. Аттоuch, Variational analysis for functions and operators, Pitman Advanced Publishing Program, Applicable Math. Series, 1984.

[3] Н. Аттоuch, А. САвот, Asymptotic stabilization of inertial gradient dynamics with time-dependent viscosity, J. Differential Equations, 263 (2017), pp. 5412-5458.

[4] H. Attouch, Z. Chbani, J. Peypouquet, P. Redont, Fast convergence of inertial dynamics and algorithms with asymptotic vanishing damping, Math. Program., (2016), https://doi.org/10.1007/s10107-016-0992-8.

[5] H. Attouch, M.-O. Czarnecki, Asymptotic behavior of coupled dynamical systems with multiscale aspects, J. Differential Equations, 248 (2010), pp. 1315-1344.

[6] H. Атtouch, P.E. Maingé, Asymptotic behavior of second-order dissipative evolution equations combining potential with non-potential effects, ESAIM: Control, Optimisation and Calculus of Variations, 17 (2010), pp. 836-857.

[7] H. Атtouch, J. Peypouquet, The rate of convergence of Nesterov's accelerated forward-backward method is actually faster than $\frac{1}{k^{2}}$, SIAM J. Optim., 26 (2016), No. 3, pp. 1824-1834.

[8] H. Attouch, J. Peypouquet, Convergence of inertial dynamics and proximal algorithms governed by maximal monotone operators, preprint available at https://arxiv.org/pdf/1705.03803.pdf, to appear in Math. Program. B, Variational Analysis in Modern Statistics.

[9] J. B. BAILlon AND H. BRÉzis, Une remarque sur le comportement asymptotique des semigroupes non linéaires, Houston J. Math. 2 (1976), No. 1, pp. 5-7.

[10] M. BALti, R. MAY, Asymptotic for the perturbed heavy ball system with vanishing damping term, Evolution Equations and Control Theory, 6 (2017), pp. 177-186.

[11] H. Bauschke, P. Combettes, Convex Analysis and Monotone Operator Theory in Hilbert spaces, CMS Books in Mathematics, Springer, (2011). 
[12] A. Beck, M. Teboulle, A fast iterative shrinkage-thresholding algorithm for linear inverse problems, SIAM J. Imaging Sci., 2 (2009), No. 1, pp. 183-202.

[13] R. I. Bot, E. R. Csetnek, Second order forward-backward dynamical systems for monotone inclusion problems, SIAM J. Control Optim., 54 (2016), pp. 1423-1443.

[14] R. I. Вот, E. R. Csetnek, Approaching monotone inclusion problems via second order dynamical systems with linear and anisotropic damping, in: V. Barbu, C. Lefter, I.I. Vrabie (Eds.), "New Trends in Differential Equations, Control Theory and Optimization", Proceedings of the 8th Congress of Romanian Mathematicians, World Scientific Publishing, Singapore, pp. 53-72.

[15] H. BRÉzis, Opérateurs maximaux monotones dans les espaces de Hilbert et équations d'évolution, Lecture Notes 5 , North Holland, (1972).

[16] H. Brézis, F.E. Browder, Nonlinear ergodic theorems, Bull. Amer. Math. Soc., 82 (1976), No. 6, pp. 959-961.

[17] A. Савот, H. Engler, S. Gadat, On the long time behavior of second order differential equations with asymptotically small dissipation, Transactions of the American Mathematical Society, 361 (2009), pp. 5983-6017.

[18] A. CAвот, H. Engler, S. Gadat, Second order differential equations with asymptotically small dissipation and piecewise flat potentials, Electronic Journal of Differential Equations, 17 (2009), pp. 33-38.

[19] А. Сhambolle, Сн. Dossal, On the convergence of the iterates of the Fast Iterative Shrinkage/Thresholding Algorithm, Journal of Optimization Theory and Applications, 166 (2015), pp. 968-982.

[20] A. Haraux, Systèmes dynamiques dissipatifs et applications, R.M.A. 17, Masson, Paris (1991).

[21] C. Imbert, Convex Analysis techniques for Hopf-Lax formulae in Hamilton-Jacobi equations, Journal of Nonlinear and Convex Analysis, Yokohama, 2 (2001), No. 3, pp. 333-343.

[22] R. MAY, Asymptotic for a second order evolution equation with convex potential and vanishing damping term, Turk. J. Math., 41 (2017), pp. 681-685.

[23] Y. Nesterov, A method of solving a convex programming problem with convergence rate $O\left(1 / k^{2}\right)$, Soviet Mathematics Doklady, 27 (1983), pp. 372-376.

[24] Z. OPIAL, Weak convergence of the sequence of successive approximations for nonexpansive mappings, Bull. Amer. Math. Soc., 73 (1967), pp. 591-597.

[25] N. PARIKh, S. Boyd, Proximal algorithms, Foundations and trends in optimization, volume 1, (2013), pp. $123-231$.

[26] G.B. PAssty, Ergodic convergence to a zero of the sum of monotone operators in Hilbert space, J. Math. Anal. Appli., 72 (1979), pp. 383-390.

[27] J. Peypouquet, Convex optimization in normed spaces: theory, methods and examples. Springer, 2015.

[28] W. Su, S. Boyd, E. J. CANDÈs, A differential equation for modeling Nesterov's accelerated gradient method: theory and insights. Neural Information Processing Systems 27 (2014), pp. 2510-2518.

Institut Montpelliérain Alexander Grothendieck, UMR 5149 CNRS, Université Montpellier, Place Eugène

Bataillon, 34095 Montpellier Cedex 5, France

E-mail address: hedy.attouch@univ-montp2.fr

Institut de Mathématiques de Bourgogne, UMR 5584, CNRS, Univ. Bourgogne Franche-Comté, 21000 Dijon, FRANCE.

E-mail address: alexandre.cabot@u-bourgogne.fr 